\title{
Identification of the Transcriptional Networks and the Involvement in Angiotensin II-Induced Injury after CRISPR/Cas9-Mediated Knockdown of Cyr61 in HEK293T Cells
}

\author{
Junjie Wang $\mathbb{D},{ }^{1}$ Dongdong Fu $\mathbb{D},{ }^{1}$ Soulixay Senouthai $\mathbb{D},{ }^{1}$ Yan Jiang $\mathbb{D},{ }^{2}$ Rentong $\mathrm{Hu}\left(\mathbb{D},{ }^{3}\right.$ \\ and Yanwu You iD 1 \\ ${ }^{1}$ Department of Nephrology, Affiliated Hospital of Youjiang Medical University for Nationalities, Baise, \\ Guangxi Zhuang Autonomous Region, China \\ ${ }^{2}$ Department of Clinical Laboratories, Affiliated Hospital of Youjiang Medical University for Nationalities, Baise, \\ Guangxi Zhuang Autonomous Region, China \\ ${ }^{3}$ Science Lab Center, Youjiang Medical University for Nationalities, Baise, Guangxi Zhuang Autonomous Region, China
}

Correspondence should be addressed to Yanwu You; youyanwu@163.com

Received 11 December 2018; Accepted 14 February 2019; Published 15 April 2019

Academic Editor: Daniela Caccamo

Copyright (C) 2019 Junjie Wang et al. This is an open access article distributed under the Creative Commons Attribution License, which permits unrestricted use, distribution, and reproduction in any medium, provided the original work is properly cited.

Background. The transcriptional networks of Cyr61 and its function in cell injury are poorly understood. The present study depicted the lncRNA and mRNA profiles and the involvement in angiotensin II-induced injury after Cyr61 knockdown mediated by CRISPR/Cas9 in HEK293T cells. Methods. HEK293T cells were cultured, and Cyr61 knockdown was achieved by transfection of the CRISPR/Cas9 KO plasmid. lncRNA and mRNA microarrays were used to identify differentially expressed genes (DEGs). Gene ontology (GO) and the Kyoto Encyclopedia of Genes and Genomes (KEGG) pathway analyses were performed to determine biofunctions and signaling pathways. RT-PCR was used to validate the microarray results. Cells were divided into four groups: control, Cyr61 knockdown, angiotensin II (Ang II) without Cyr61 knockdown, and Ang II with Cyr61 knockdown. CCK8, western blotting, and flow cytometry analysis were carried out to dissect cellular function. Results. A total of 23184 lncRNAs and 28264 mRNAs were normalized. 26 lncRNAs and 212 mRNAs were upregulated, and 74 lncRNAs and 233 mRNAs were downregulated after Cyr61 knockdown. Analysis of cellular components, molecular functions, biological processes, and regulatory pathways associated with the differentially expressed mRNAs revealed downstream mechanisms of the Cyr61 gene. The differentially expressed genes were affected for small cell lung cancer, axon guidance, Fc gamma R-mediated phagocytosis, MAPK signaling pathway, focal adhesion, insulin resistance, and metabolic pathways. In addition, Cyr61 expression was increased in accordance with induction of cell cycle arrest and apoptosis and inhibition of cell proliferation induced by Ang II. Knockdown of Cyr61 in HEK293T cells promoted cell cycle procession, decreased apoptosis, and promoted cell proliferation. Conclusions. The Cyr61 gene is involved in Ang II-induced injury in HEK293T cells. Functional mechanisms of the differentially expressed lncRNAs and mRNAs as well as identification of metabolic pathways will provide new therapeutic targets for Cyr61-realated diseases.

\section{Introduction}

Cysteine-rich protein 61 (Cyr61), also known as CCN1, was identified as secreted protein members of the CCN family. Cyr61 which is produced and secreted by endothelial cells, fibroblasts, and smooth muscle cells is a component of the extracellular matrix [1]. Cyr61 has the ability to regulate multiple signaling pathways and play an important role in different biological processes. In vitro, $\mathrm{CCN}$ proteins regulate the functions of cells, such as adhesion, migration, proliferation, differentiation, and survival, as well as induce cell apoptosis and extracellular matrix remodeling of different cell types. In vivo, it also plays a vital role in vascular and bone development and angiogenesis [2]. Furthermore, Cyr61 has 
highlighted the pivotal role this molecule can play in regulating the immune-surveillance process and it has emerged as an important partner when targeting components of the infectious or chronic inflammatory disease processes such as atherosclerosis or rheumatoid arthritis [3-5]. Additionally, several lines of evidence have shown that overexpression of Cyr61 is involved in the cancer process such as osteosarcoma [6], ovarian tumor [7], and breast adenocarcinoma [8]. Interestingly, Cyr61 was also found to be downregulated in prostate cancer and non-small cell lung carcinoma $[9,10]$. This can be ascribed to its direct binding to different integrins in different cell types and a variety of environments [11]. Hence, Cyr61 may play a pivotal role and serve as a potential prognostic indicator or therapeutic target in a variety of diseases.

The mammalian genome is pervasively transcribed, producing a large number of noncoding RNAs (ncRNAs), including long noncoding RNAs (lncRNAs), which are defined as transcripts longer than 200 nucleotides [12, 13]. Studies have found that only one-fifth of transcription across the human genome is associated with protein-coding genes, indicating that there are at least four times more long noncoding than coding RNA sequences [14]. IncRNAs play an important role in the regulation of thousands of proteinencoding genes via various mechanisms, although these are not translated into proteins [15]. Transcriptome profiling has been carried out in various genes and conditions in order to characterize their functions and underlying mechanisms [16-19]. Unfortunately, studies of Cyr61 functional recovery have not yet been able to highlight its transcriptional networks. No study so far has reported using the gene chip array to find out about the differentially expressed lncRNAs and mRNAs associated to Cyr61.

As one of the earliest and most extensively studied hormonal systems, the renin-angiotensin system (RAS) which is involved in many physiological and pathological processes is an atypical hormonal system in several ways [20,21], and the new components and functions of the RAS have still not been unraveled [22]. The RAS has powerful effects including the control of blood pressure and sodium homeostasis as well as fluid through integrated actions in the kidney, the cardiovascular system, and the central nervous system. Along with its impact on blood pressure, the RAS also affects others pathological processes including inflammation and immune responses [23]. Research on the RAS has contributed significantly to advances in the understanding and treatment of cardiovascular diseases [24]. As one of the major bioactive products of the RAS, angiotensin II (Ang II) has a well-known role in cardiovascular regulation and is a key regulator of renal inflammation and fibrosis as well as blood pressure and renal hemodynamics [25-27]. Several pathological effects are caused by the Ang II-induced TGF- $\beta$ pathway which is a potent inflammatory as well as fibrotic and apoptotic cytokine [28, 29]. In addition, Ang II participates in cell proliferation, matrix degradation, inflammation, and apoptosis by activating a multiplicity of signaling pathways [30,31].

In this study, the lncRNA and mRNA profiles were determined after Cyr61 knockdown mediated by CRISPR/Cas9 in
TABLE 1: The guide RNAs sequences.

\begin{tabular}{lcc}
\hline gRNA & Name & Sequence \\
\hline Cyr61-gRNA1 & Cyr61-A & GTTGTCATTGGTAACTCGTG \\
Cyr61-gRNA2 & Cyr61-B & ATGCGGTTCCGCTGCGAAGA \\
Cyr61-gRNA3 & Cyr61-C & CAAGTACTGCGGTTCCTGCG \\
\hline
\end{tabular}

HEK293T cells. The signal transduction and metabolic pathways associated with these differentially expressed lncRNA and mRNA were analyzed. The effect of Cry61 on Ang IIinduced cell injury in HEK293T cells was also analyzed. The results provide the functional mechanisms of Cry61 as well as potential new targets for Cyr61-associated diseases.

\section{Materials and Methods}

2.1. Cell Lines and Cell Culture. The HEK293T cell line (which was purchased from the Molecular Microbiology and Immunology, Keck School of Medicine, University of Southern California) was cultured in Dulbecco's modified Eagle's medium (DMEM, Gibco, Su Zhou, USA) in the presence of $10 \%$ fetal bovine serum (FBS, Lanzhou Minhai BioEngineering, Gansu, China). All of the cells were maintained at $37^{\circ} \mathrm{C}$ in a humidified atmosphere with $5 \% \mathrm{CO}_{2}$.

2.2. Knockdown of Cyr61 Gene Expression. The guide RNA sequences used are shown in Table 1. Cyr61 CRISPR/Cas9 $\mathrm{KO}$ plasmid, homology-directed repair (HDR) transfection plasmid, and UltraCruz Transfection Reagent were purchased from Santa Cruz Biotechnology (USA), and gene knockdowns were performed according to the manufacturer's protocol. The HEK293T cells were seeded in 6-well plates at $1.5 \times 10^{5}-2.5 \times 10^{5}$ cells $/ 3 \mathrm{~mL}$ per well, and the serum-free medium was changed after $70 \%$ confluence was achieved by routine culture. $1 \mu \mathrm{g}$ of each of Cyr61 CRISPR/Cas9 KO plasmid and HDR transfection plasmid and $10 \mu \mathrm{L}$ of UltraCruz reagents were added to $300 \mu \mathrm{L}$ of serum-free and antibiotic-free medium and in 6-well plates for incubation at room temperature for $10 \mathrm{~min}$, followed by addition of antibiotic-free $10 \%$ FBS-containing media to a final volume of $2 \mathrm{~mL}$. Wild-type HEK293T cells were treated with different concentrations of puromycin, and the results showed that the lowest inhibitory concentration to the HEK293T cells after $7 \mathrm{~d}$ of treatment was $8 \mu \mathrm{g} / \mathrm{mL}$ and this concentration was subsequently used for screening. At $48 \mathrm{~h}$ posttransfection, the culture medium was replaced with $8 \mu \mathrm{g} / \mathrm{mL}$ puromycin-containing DMEM medium for screening the cells.

2.3. Classification of Cells. The HEK293T cells were divided into four groups: (1) control group, nontransfected cells, (2) Cyr61-downregulated group, the third generation incubated after transfection, (3) Ang II group, nontransfected cells treated with Ang II $\left(10^{-7} \mathrm{~mol} / \mathrm{L}\right)$, and (4) Cyr61 downregulated + Ang II group, transfected cells treated with Ang II $\left(10^{-7} \mathrm{~mol} / \mathrm{L}\right)$. Cyr61 expression in the control group and Cyr61-downregulated group were identified by western blotting, and these were subjected to microarray analysis. Cell 
TABLE 2: The RNA quality test results.

\begin{tabular}{lcccccc}
\hline Sample name & Sample code & $\mathrm{OD}_{260} / \mathrm{OD}_{280} \geq 1.8$ & $\mathrm{OD}_{260} / \mathrm{OD}_{230} \geq 1.5$ & Total of RNA & RIN $>6$ & Result \\
\hline $2402-1$ & $1 \mathrm{H} 617022202-01 \mathrm{~A}$ & 2.02 & 2.12 & 53.6 & 10.0 & Pass \\
$2402-2$ & $1 \mathrm{H} 617022202-02 \mathrm{~A}$ & 2.02 & 2.02 & 55.8 & 10.0 \\
$2402-3$ & $1 \mathrm{H} 617022202-03 \mathrm{~A}$ & 2.01 & 2.15 & 57.0 & 10.0 & Pass \\
$2403-1$ & $1 \mathrm{H} 617022202-04 \mathrm{~A}$ & 2.01 & 2.12 & 66.5 & 9.9 & Pass \\
$2403-2$ & $1 \mathrm{H} 617022202-05 \mathrm{~A}$ & 2.01 & 2.07 & 71.4 & 10.0 \\
$2403-3$ & $1 \mathrm{H} 617022202-06 \mathrm{~A}$ & 2.02 & 2.13 & 64.6 & 9.9 & Pass \\
\hline
\end{tabular}

RIN values were detected using the Agilent RNA 6000 Nano Assay, and the integrity of 18s and 28s (for eukaryotes) in the RNA samples was detected to determine the integrity of RNA. RIN is between 0 and 10, with a full score of 10. RIN values of more than 6 indicate good sample integrity and can be used in ChIP experiments.

proliferation, apoptosis, and cell cycle assays were performed for all the groups.

\subsection{RNA Extraction and Quality Control. Total RNA was} extracted from each sample by soaking the samples in TRIzol Reagent (Invitrogen, Grand Island, NY, USA) in accordance with the manufacturer's instructions. RNA quantity and quality were measured using a NanoDrop ND-1000, and RNA integrity was assessed by standard denaturing agarose gel electrophoresis. RNA quality test results are shown as Supplemental Material (Table 2). For spectrophotometer analysis, only samples with $\mathrm{OD}_{260} / \mathrm{OD}_{280}$ ratio $\geq 1.8$ and $\mathrm{OD}_{260} / \mathrm{OD}_{230}$ ratio $\geq 1.5$ were acceptable for further analysis.

2.5. Identification of Differentially Expressed $\operatorname{lncRNAs}$ and $m R N A s$. The mRNA-lncRNA Affymetrix microarrays (raw data) were used in this study. Limma (linear models for microarray data) package in $\mathrm{R}$ was used to identify differentially expressed lncRNAs and mRNAs between the 2 groups through use of the $t$-test. Fold change $>1$ and $P<0.05$ were regarded as the criteria for differential expression.

2.6. Gene Ontology (GO) and Pathway Enrichment Analysis. $\mathrm{GO}$ analysis is frequently used in functional enrichment studies of large-scale genes. Kyoto Encyclopedia of Genes and Genomes (KEGG) enrichment analysis was performed to analyze the biological pathways, involving the differentially expressed mRNAs. In the current study, DAVID (the database for Annotation Visualization and Integrated Discovery) software was used to investigate the functional enrichment condition for the up- and downregulated differentially expressed mRNAs. $P<0.05$ was selected as the threshold.

2.7. Quantitative Real-Time PCR Validation. To validate the microarray data, we selected the top 3 most significant upregulated (FRMD1, SERPINF1, and FEZ1) and the top 3 most significant downregulated (PRR21, REG3G, and ACAT2) mRNAs as well as the top 3 most significant upregulated (RP11-659F24.1, RP11-966I7.4, and LAMB2P1) and the top 3 most significant downregulated (ANKRD30BL, CH17360D5.2, and SOX2-OT) lncRNAs from mRNAs and lncRNAs that were expressed aberrantly. Briefly, $2 \mu \mathrm{g}$ of total RNA from each sample was used for the synthesis of firststrand cDNA using a FastKing RT Kit (Tiangen, Beijing, China) according to the manufacturer's protocol. Following first-strand cDNA synthesis, a PCR reaction was carried out in a $20 \mu \mathrm{L}$ reaction volume containing $\times 1$ SuperReal PreMix Plus, $0.6 \mu \mathrm{L}$ of each specific forward and reverse primer, and $2 \mu \mathrm{L}$ of cDNA template. PCR assays were set at an initial denaturation step at $95^{\circ} \mathrm{C}$ for $3 \mathrm{~min}$, followed by 40 cycles of $95^{\circ} \mathrm{C}$ for $10 \mathrm{~s}$ and $60^{\circ} \mathrm{C}$ for $30 \mathrm{~s}$ in a LightCycler ${ }^{\circledR} 96$ system (Roche, Switzerland). Relative changes in gene expression were calculated using the $2^{-\Delta \Delta C t}$ method with glyceraldehyde 3-phosphate dehydrogenase (GAPDH) as a reference gene as described previously $[32,33]$. Each qPCR was carried out in triplicate, with each triplicate data point repeated 3 times. For the triplicates, only samples that differed by $<0.3 \mathrm{Ct}$ values were used in the final calculations. Less than about $5 \%$ of samples fell outside this range. In addition, standard curves were generated for each gene using a 50 -fold dilution range. The data shown represented the means of three experiments. The primers for each gene are listed in Table 3.

2.8. Western Blotting Analysis. The HEK293T cells were harvested and washed twice with PBS, lysed in ice-cold radio-immunoprecipitation assay buffer (RIPA, Beyotime Biotechnology, Shanghai, China) with a freshly added $0.01 \%$ protease inhibitor cocktail (CWBiotech, Beijing, China), and incubated on ice for 30 minutes. Protein concentration was measured by bicinchoninic acid (BCA) protein assay kit (Beyotime Biotechnology, Shanghai, China). A total of $50 \mu \mathrm{g}$ of protein was subjected to electrophoresis using SDS-PAGE and transferred by electrophoresis onto a nitrocellulose membrane (GE Healthcare, Freiburg, Germany). Blots were visualized using enhanced chemiluminescence (ECL, Millipore, Billerica, MA, USA) after antibody binding. The antibody against Cyr61 was purchased from Santa Cruz Biotechnology (USA); antibodies against GAPDH were purchased from Cell Signaling Technology Biotech (Danvers, MA, USA). The bands were quantified by their densitometry with ImageJ software (NIH, USA).

2.9. Cell Proliferation Assay. Cell proliferation was performed using Cell Counting Kit-8 (CCK-8, Solarbio Life Sciences, Beijing, China) assay. The HEK293T cells were seeded in 96-well plates at a density of $5 \times 10^{3}$ cells per well and cultured at $37^{\circ} \mathrm{C}$ in $5 \% \mathrm{CO}_{2}$ for 12 hours and treated with Ang II $\left(10^{-7} \mathrm{~mol} / \mathrm{L}\right)$ [34]. After incubation for $0,12,24$, and $48 \mathrm{~h}$, $10 \mu \mathrm{L}$ CCK- 8 solution was added to each well and incubated for another 1 to 4 hours. The absorbance was measured in the 
TABle 3: Primers for qRT-PCR.

\begin{tabular}{lcc}
\hline Name & Forward primer $\left(5^{\prime}-3^{\prime}\right)$ & Reverse primer $\left(5^{\prime}-3^{\prime}\right)$ \\
\hline GAPDH & AACTTTGGCATTGTGGAAGG & GGATGCAGGGATGATGTTCT \\
RP11-659F24.1 & CTCGGCTCACTGAAAACTCC & TTCAACACCGTGCCTTCATA \\
RP11-966I7.4 & TCCTGGGGCACTAATAGCAG & TCTTACGAGCGTCTCCACCT \\
LAMB2P1 & CCTTTCTGGTTCGGCCTCAG & GGATCCTCTCAGGGATGACA \\
ANKRD30BL & CCAGAAGGAACATCTACAGGAACACC & CAAGCGTGCAGCCTCGTCAG \\
CH17-360D5.2 & CCGCCATACAGATACGAAGCCAAG & ATCCTCTCCAGGTAGCCACTTGTG \\
SOX2-OT & AGGTCTTGGAGGCTGGTGTAAGG & ACCATGTGAAGGAGCTTGCAGTTC \\
FRMD1 & GCACTACGTGGAAAACGGAAG & GAGTGTGGCTCGAAGTACCTC \\
SERPINF1 & TTCAAAGTCCCCGTGAACAAG & GAGAGCCCGGTGAATGATGG \\
FEZ1 & CCACTGGTGAGTCTGGATGAA & CGGAAGAAAAATTCTCAAGCTCG \\
PRR21 & CTCCGCGTTCTTATCTGTG & GGACAGGAGGCTGAGAAGTT \\
REG3G & GGTGAGGAGCATTAGTAACAGC & CCAGGGTTTAAGATGGTGGAGG \\
ACAT2 & GCGGACCATCATAGGTTCCTT & ACTGGCTTGTCTAACAGGATTCT \\
\hline
\end{tabular}

multimode microplate reader TriStar LB 941 (Berthold Technologies, Bad Wildbad, Germany) at $450 \mathrm{~nm}$. Each experiment was performed in triplicate.

2.10. Cell Apoptosis Assay. Apoptosis was determined by using a flow cytometer (FACSCanto II, BD Biosciences, San Jose, CA, USA), and an Annexin V fluorescein isothiocyanate (FITC)/propidium iodide (PI) double-stain assay was performed in accordance with the manufacturer's protocol (FITC Annexin V/PI, BD Biosciences, San Diego, USA). After incubation for 48 hours, each supernatant was collected in a centrifuge tube and each group of cells was washed three times with phosphate-buffered saline (PBS), trypsinized, centrifuged ( $400 \times g$ at room temperature) for $5 \mathrm{~min}$, adjusted to $5 \times 10^{4}$ cells $/ \mathrm{mL}$, and suspended in binding buffer containing Annexin V-FITC and PI. After incubation for a further $15 \mathrm{~min}$ at room temperature in the dark, the fluorescent intensity was measured using a flow cytometer. Each experiment was performed in triplicate.

2.11. Cell Cycle Assay. For cell cycle analysis, each group of cells was harvested after $48 \mathrm{~h}$. Cell cycle analysis was conducted with the cell cycle detection kit (KeyGen, China) according to the manufacturer's instructions. Briefly, cells were harvested by regular trypsin digestion and rinsed with PBS. After fixation in $70 \%$ ethanol overnight at $-20^{\circ} \mathrm{C}$, cells were rinsed again in PBS and stained in a staining solution containing PI and RNase A. Flow cytometry (Canto II, BD Biosciences, USA) was employed to analyze the cellular DNA content. Each treatment was performed in triplicate.

2.12. Statistical Analysis. The results are presented as the mean value \pm S.D. using SPSS23. One-way analysis of variance (ANOVA) was used among diverse groups, and independent samples were analyzed by Student's $t$-test when appropriate. A value of $P<0.05$ was regarded as statistically significant.

To determine if DEGs overlapped with or were significantly enriched with a specific gene set, 28264 expressed genes in our samples were used as background for gene ontology (GO) and Kyoto Encyclopedia of Genes and Genomes (KEGG) pathway analyses assessed by DAVID.

\section{Results}

3.1. The Expression Levels of Cyr61 Protein in HEK293T Cells. To examine the biological function of Cyr61 in HEK293T cells, the expression of Cyr61 was knocked down in HEK293T cells by the CRISPR/Cas9 KO plasmid, and three separate CRISPR gRNA sequences targeted to the Cyr61 gene were designed. The HEK293T cells were transfected with CRISPR/Cas9 vectors containing each of the three target sequences. After screening by puromycin, one homozygous was found by western blotting analysis. As shown in Figure 1, the Cyr61 protein expression levels were significantly decreased in HEK293T cells from $1.03 \pm 0.024$ to $0.36 \pm 0.021$ and from $1.06 \pm 0.018$ to $0.28 \pm 0.023$ (Cyr61/GAPDH, respectively) after knockdown from control levels.

3.2. Differential Gene Expression Analysis between Experimental and Control Groups. 100 differentially expressed lncRNAs with a fold change cutoff of 1.0 (26 upregulated and 74 downregulated, $P<0.05$ ) were identified from the lncRNA microarrays of the Cyr61 experimental and control groups. Volcano plots were constructed to identify differences in the lncRNAs. The top 10 most significant up- and downregulated lncRNAs are shown in Table 4. RP11-659F24.1 and ANKRD30BL were the most up- and downregulated $\operatorname{lncRNA}$ transcript with a fold change of 2.87 and 2.13 , respectively. The volcano plots display the expression profiles for all detected lncRNAs (Figure 2(a)).

446 differentially expressed mRNAs were also identified by using mRNA microarrays with a fold change cutoff of 1.0 (212 upregulated and 223 downregulated, $P<0.05$ ) in Cyr61 knocked down HEK293T cells when compared with controls. The top 10 most significant up- and downregulated mRNAs are shown in Table 5. FRMD1and PRR21 were the most up- and downregulated mRNA transcripts with the fold change of 2.7 and 2.02, respectively. The volcano plots 


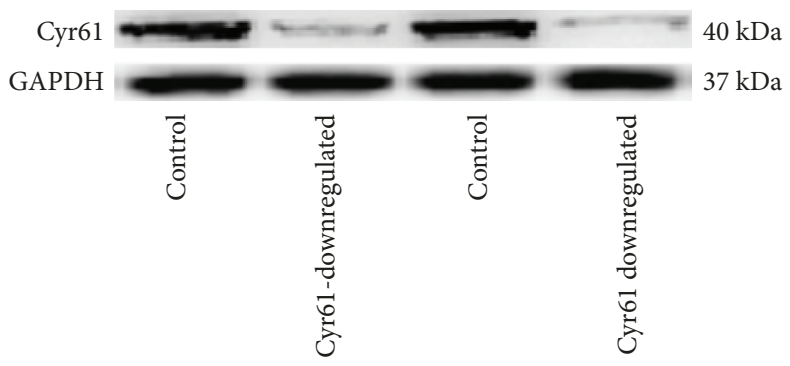

(a)

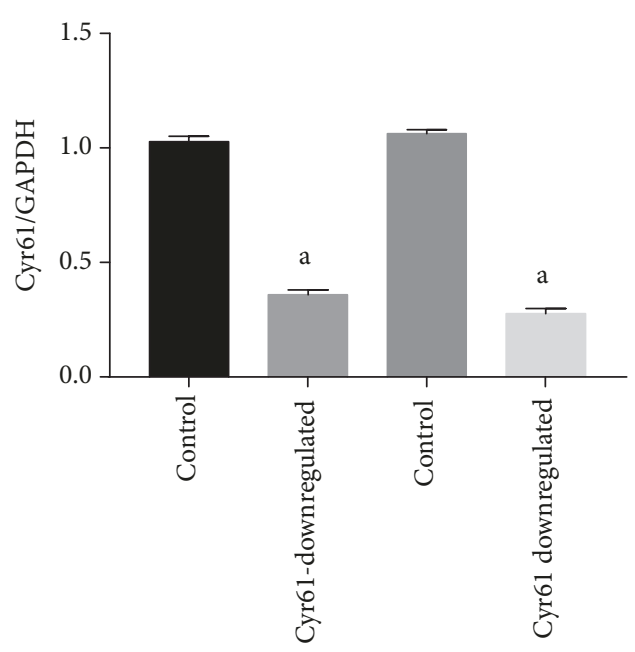

(b)

FIGURE 1: The expression of Cyr61 protein by transfection of CRISPR/Cas9 KO plasmid. The expression of Cyr61 protein was confirmed by western blotting. The Cyr61 protein expression levels were significantly decreased in HEK293T cells where the Cyr61 gene was knocked down when compared with those of the controls $\left({ }^{\mathrm{a}} \mathrm{P}<0.05\right)$. The data represent mans $\pm \mathrm{SD}$ from 3 independent experiments.

TABLE 4: The top 10 most significant up- and downregulated lncRNAs.

\begin{tabular}{|c|c|c|c|c|c|}
\hline \multirow{2}{*}{$P$ value } & \multirow{2}{*}{ Gene symbol } & \multicolumn{2}{|c|}{ Fold change $(\log \mathrm{FC})$} & \multirow{2}{*}{ Gene symbol } & \multirow{2}{*}{$P$ value } \\
\hline & & Upregulation & Downregulation & & \\
\hline 0.0062 & RP11-659F24.1 & 2.8653 & -2.1347 & ANKRD30BL & 0.0026 \\
\hline 0.0496 & RP11-966I7.4 & 2.6712 & -2.0459 & CH17-360D5.2 & 0.0015 \\
\hline $2.44 E-06$ & LAMB2P1 & 2.3529 & -1.5879 & SOX2-OT & 0.0010 \\
\hline $1.14 E-05$ & CECR5-AS1 & 1.8255 & -1.5293 & CTD-3099C6.11 & 0.0125 \\
\hline $5.98 E-06$ & RP11-617F23.1 & 1.7205 & -1.5262 & RP11-227G15.3 & 0.0324 \\
\hline $7.29 E-06$ & RP11-49I11.1 & 1.5183 & -1.5095 & RP1-290I10.3 & 0.0011 \\
\hline $3.75 E-05$ & LINC00505 & 1.4474 & -1.5090 & HOXA11 & $1.25 E-05$ \\
\hline 0.0005 & LINC01021 & 1.4186 & -1.5085 & FLJ33534 & 0.0024 \\
\hline $2.64 E-05$ & RP11-395B7.7 & 1.3960 & -1.4651 & LINC00948 & 0.0190 \\
\hline 0.0003 & LINC01021 & 1.3026 & -1.4335 & DLEU2 & $2.26 E-05$ \\
\hline
\end{tabular}

display the expression profiles for all detected mRNAs (Figure 2(b)). The heat map is shown in Figure 2(c).

3.3. Gene Ontology Analysis. The 446 differentially expressed mRNAs underwent Gene Ontology (GO) analyses by using the DAVID software. GO analysis of the differentially expressed genes was performed to determine the gene product attributes in biological processes, cellular components, and molecular functions. $P<0.05$ denoted the significance of GO term enrichment in the differentially expressed genes, and the lower the $P$ value, the more significant the GO term. The top 10 biological processes with the highest $P$ values are listed in Table 6 and Figure 3(a), and the most significant process is related to "regulation of cell migration." The top 10 cellular components with the highest $P$ values are shown in Table 7 and Figure 3(b), and the most significant one is the "cytosol." The top 10 molecular functions terms with the highest $P$ values are displayed in
Table 8 and Figure 3(c), and the most significant one is "poly (A) RNA binding."

3.4. KEGG Pathway Analysis. Pathway analysis is a functional analysis that maps genes to the KEGG pathways. The target genes for differentially expressed mRNAs were mapped onto signaling pathways for KEGG pathway analysis (Table 9 and Figure 3(d)). The differentially expressed genes were affected for small cell lung cancer, axon guidance, Fc gamma R-mediated phagocytosis, MAPK signaling pathway, focal adhesion, insulin resistance, and metabolic pathways. The results revealed that the differentially expressed gene annotation for metabolic pathway and MAPK signaling pathway are the top 2 signaling pathways, while the small lung cancer is the most significant.

3.5. Validation of $\operatorname{lncRNA}$ Expression by RT-qPCR. To validate the up- and downregulation of Cyr61 changes of mRNA and lncRNA expression detected by microarray, six mRNAs 


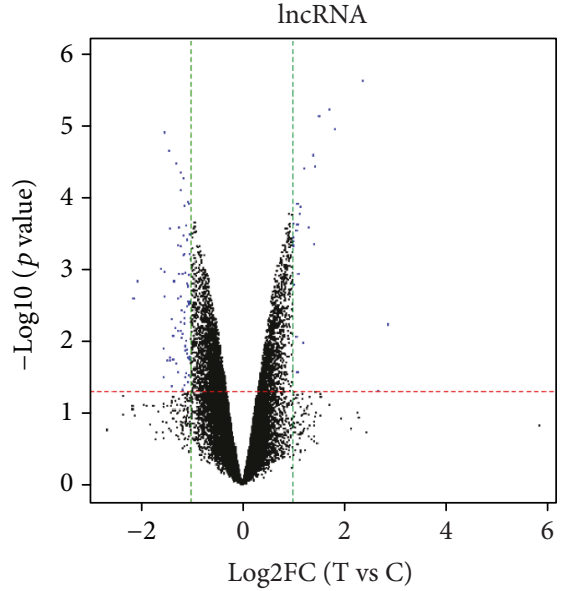

(a)

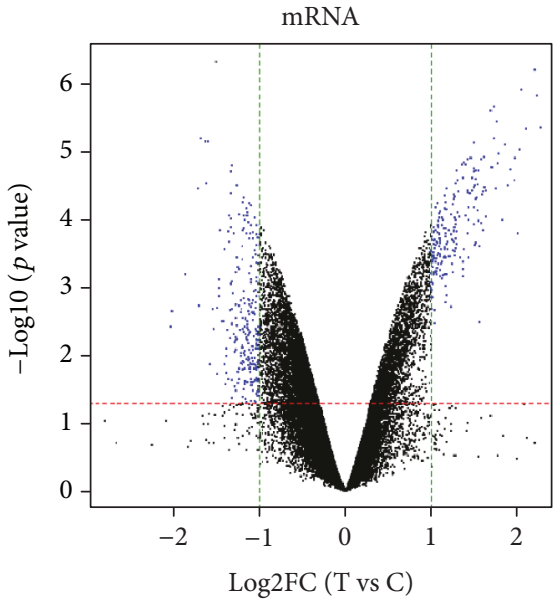

(b)

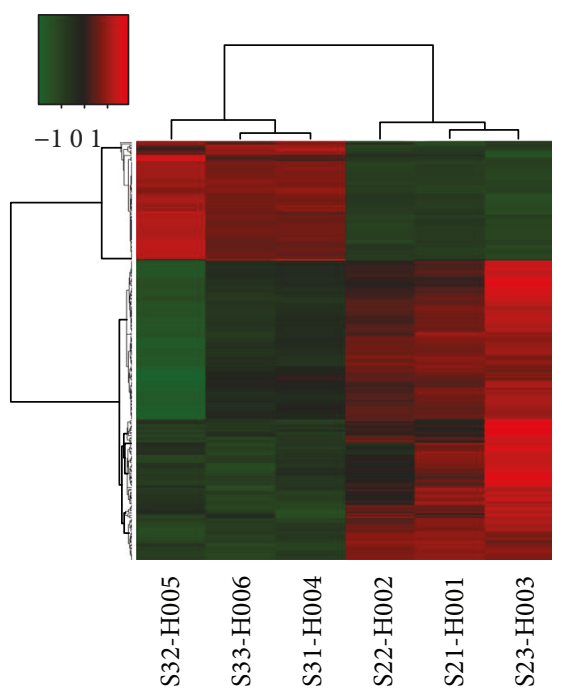

(c)

FiguRE 2: Volcano plots and heat map. Volcano plots of all the detected lncRNAs (a) and mRNAs (b) in the control group and downregulated Cyr61 group. The red- and green-dotted lines represent $P$ values and multiple screening thresholds, respectively. The blue plots represent significantly changed genes with $\geq 1.0$-fold change and $P$ values of 0.05 . The heat map displayed differentially expressed mRNAs. (c). Each column represents a sample, and each row represents a dysregulated RNA transcript. The red and green stripes imply that the RNA was upregulated and downregulated, respectively, in the downregulated Cyr61 sample.

TABLE 5: The top 10 most significant up- and downregulated mRNAs.

\begin{tabular}{|c|c|c|c|c|c|}
\hline \multirow{2}{*}{$P$ value } & \multirow{2}{*}{ Gene symbol } & \multicolumn{2}{|c|}{ Fold change (log FC) } & \multirow{2}{*}{ Gene symbol } & \multirow{2}{*}{$P$ value } \\
\hline & & Upregulation & Downregulation & & \\
\hline $5.10 E-07$ & FRMD1 & 2.6979 & -2.020 & PRR21 & 0.0039 \\
\hline $4.62 E-07$ & SERPINF1 & 2.4507 & -2.008 & REG3G & 0.0022 \\
\hline $4.47 E-06$ & FEZ1 & 2.2722 & -1.851 & ACAT2 & 0.0006 \\
\hline $1.50 E-06$ & CRABP2 & 2.2282 & -1.701 & PIGW & $3.57 E-05$ \\
\hline $6.45 E-07$ & G0S2 & 2.2042 & -1.698 & RBM19 & 0.0019 \\
\hline $4.76 E-06$ & $\mathrm{CDA}$ & 2.1142 & -1.674 & RPL27A & $6.55 E-06$ \\
\hline $1.29 E-06$ & TXNIP & 2.0500 & -1.623 & ATF5 & $7.15 E-06$ \\
\hline $9.71 E-06$ & PADI2 & 2.0345 & -1.610 & ADI1 & $2.93 E-05$ \\
\hline 0.0001 & SERPINF1 & 2.0129 & -1.581 & ADI1 & $7.22 E-06$ \\
\hline $1.27 E-05$ & CRABP2 & 1.9778 & -1.565 & UBAP2L & 0.0138 \\
\hline
\end{tabular}


TABle 6: The top 10 biological process terms with the highest $P$ values.

\begin{tabular}{lcrr}
\hline GO ID & Gene set name & Count & $P$ value \\
\hline GO:0030336 & Negative regulation of cell migration & 9 & 0.0009 \\
GO:0045892 & Negative regulation of transcription, DNA-templated & 22 & 0.0023 \\
GO:0006488 & Dolichol-linked oligosaccharide biosynthetic process & 4 & 0.0036 \\
GO:0071333 & Cellular response to glucose stimulus & 6 & 0.0048 \\
GO:0006397 & mRNA processing & 11 & 0.0049 \\
GO:0098609 & Cell-cell adhesion & 5 & 0.0053 \\
GO:0000381 & Regulation of alternative mRNA splicing, via spliceosome & 4 & 0.0083 \\
GO:0048843 & Negative regulation of axon extension involved in axon guidance & 0.0171 \\
GO:0045010 & Actin nucleation & 3 & 0.0180 \\
GO:0006405 & RNA export from nucleus & 5 & 0.0290 \\
\hline
\end{tabular}

(FRMD1, SERPINF1, FEZ1, PRR21, REG3G, and ACAT2) and six lncRNAs (RP11-659F24.1, RP11-966I7.4, LAMB2P1, ANKRD30BL, CH17-360D5.2, and SOX2-OT) were selected. Their expression was examined with RT-qPCR. We observed a good agreement across the two methods. The results of the RT-qPCR were similar to those obtained from the microarray. In both microarrays and RT-qPCR, the expression levels of the mRNAs FRMD1, SERPINF1, and FEZ1 and lncRNAs RP11-659F24.1, RP11-966I7.4, and LAMB2P1 were upregulated, and the expression levels of the mRNAs PRR21, REG3G, and ACAT2 and lncRNAs ANKRD30BL, CH17360D5.2, and SOX2-OT were downregulated after knocking down the Cyr61 gene. This validation indicated good reproducibility and reliability of the observed changes in expression detected by mRNA and lncRNA microarrays (Figure 4).

3.6. Downregulation Cyr61 Promotes Proliferation of HEK293T Cells. To investigate the role of Cyr61 knockdown on the growth of HEK293T cell lines, we treated the HEK293T cells with Ang II at various time points (0, 12, 24 , and $48 \mathrm{~h}$ ) after knocking down the Cyr61 gene and then performed the CCK-8 assay to examine the proliferation of HEK293T cells. Knockdown of the Cyr61 gene increased the cell proliferation of HEK293T cells significantly, and Ang II inhibited proliferation when compared with control groups. The proliferation rate of Ang II with Cyr61 knockdown groups was significantly increased compared to cells treated with Ang II without Cyr61 knockdown. There was no significant difference between controls and Ang II with Cyr61 knockdown (Figure 5). Taken together, these data suggest a pro-proliferative role of downregulation of the Cyr61 gene and an antiproliferative role of Ang II in HEK293T cells.

3.7. Downregulation of Cyr61 Inhibits Apoptosis of HEK293T Cells. In order to investigate the role of Cyr61 on the apoptosis of HEK293T cells, we treated the HEK293T cells with Ang II after knocking down the Cyr61 gene for $48 \mathrm{~h}$, and then cells were stained with Annexin V-FITC and PI, followed by flow cytometry analysis. Under both conditions, the apoptosis rate was decreased significantly in Cyr61 knockdown groups $(5.14 \pm 1.04 \%)$ and significant cell apoptosis was observed in the Ang II groups $(32.50 \pm 2.95 \%)$ compared with controls $(12.92 \pm 2.07 \%)$. The percentage of Annexin V-FITC for Ang
II with Cyr61 knockdown groups were significantly decreased $(16.19 \pm 1.40 \%)$ compared with Ang II without Cyr61 knockdown $(32.50 \pm 2.95 \%)$. However, there was no significant difference between controls $(12.92 \pm 2.07 \%)$ and Ang II with Cyr61 knockdown (16.19 $\pm 1.40 \%$; Figure 6). Taken together, these data suggest a proliferative role after downregulation of the Cyr61 gene and an antiproliferative role of Ang II in HEK293T cells.

3.8. Downregulation of Cyr61 Promotes Cell Cycle Progression of HEK293T Cells. Having found the interaction between Cyr61 and Ang II on the apoptosis and proliferation in HEK293T cells, the interaction between these on the cell cycle was investigated by treating HEK293T cells with Ang II for $48 \mathrm{~h}$ after knocking down the Cyr61 gene. Cells were stained with PI and collected to be examined on a flow cytometer. An increase in the percentage of cells in the G2/M phase $(14.57 \pm 0.51 \%)$ and a marked decrease in the percentage of cells in the S phase $(34.74 \pm 0.30 \%)$ in Cyr61 knockdown groups compared with the other groups were seen. In addition, a decrease in the percentage of cells in the G2/M phase $(3.45 \pm 0.39 \%)$ and a marked increase in the percentage of cells in the S phase $(52.78 \pm 1.11 \%)$ in Ang II groups were also seen. There was no significant difference between controls (G2/M phase: $10.16 \pm 0.63 \%, \mathrm{~S}$ phase: $42.36 \pm 1.88$ $\%)$ and Ang II with Cyr61 knockdown groups (G2/M phase: $10.42 \pm 0.47 \%$, S phase: $41.40 \pm 1.01 \%$; Figure 7). Downregulation of Cyr61 promoted the cell cycle progression, and treatment with Ang II inhibited the cell cycle progression.

3.9. Ang II Promotes Cyr61 Expression in HEK293 T Cells. To investigate whether Ang II induces Cyr61 expression in 293 cells, we incubated HEK293T cells for 48 hours in DMEM with or without Ang II. Cyr61 transcript levels as determined by western blots were elevated $(1.56 \pm 0.031$, Cyr61/GAPDH) in HEK293T cells exposed to Ang II compared with controls $(0.98 \pm 0.026$, Cyr61/GAPDH). Furthermore, the Cyr61 protein transcript levels of HEK293T cells which had the Cyr61 gene knocked down was elevated significantly $(0.72 \pm 0.027$, Cyr61/GAPDH) after treating with Ang II compared to cells not treated with Ang II (0.48 \pm 0.032 , Cyr61/GAPDH; Figure 8). 


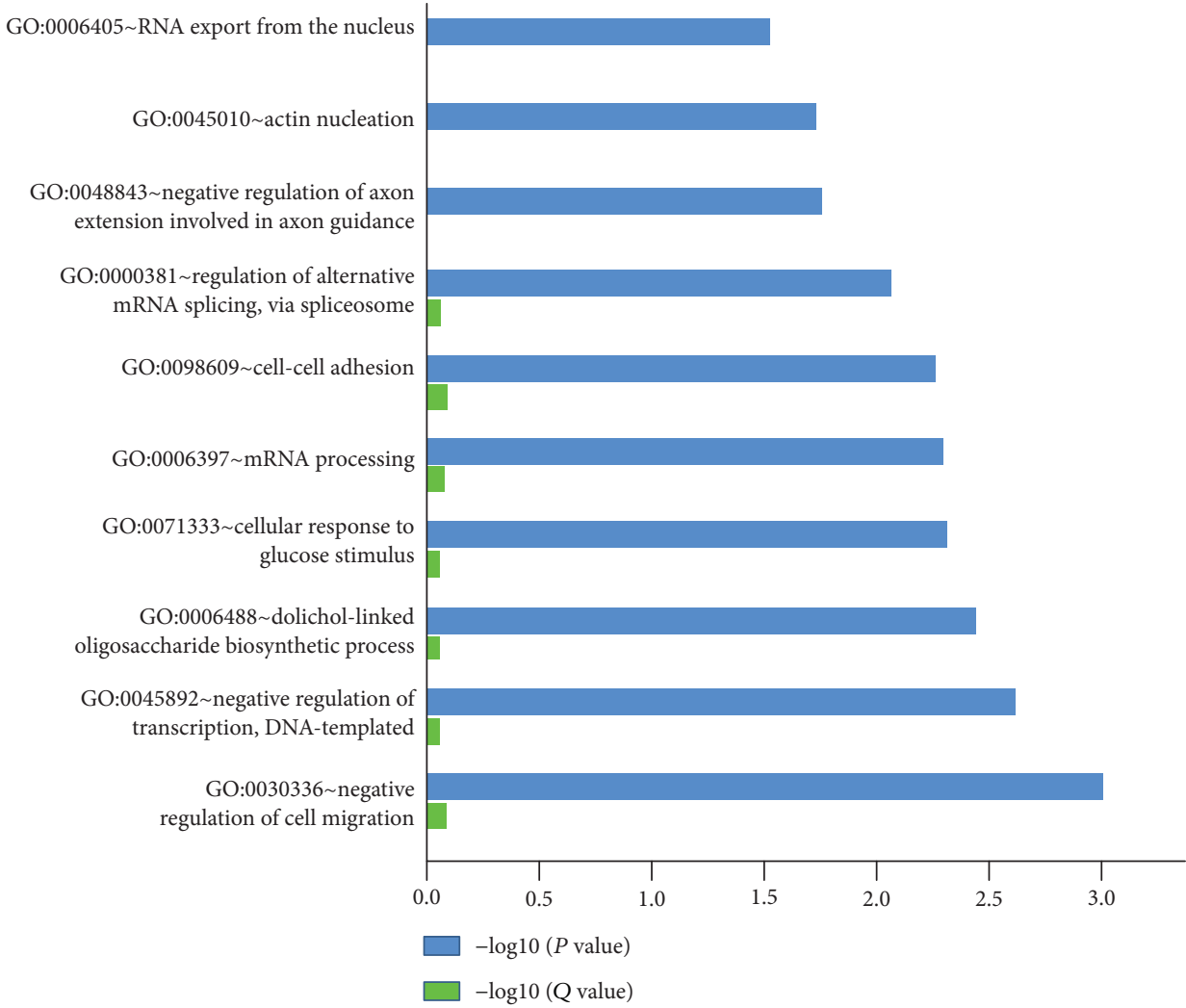

(a)

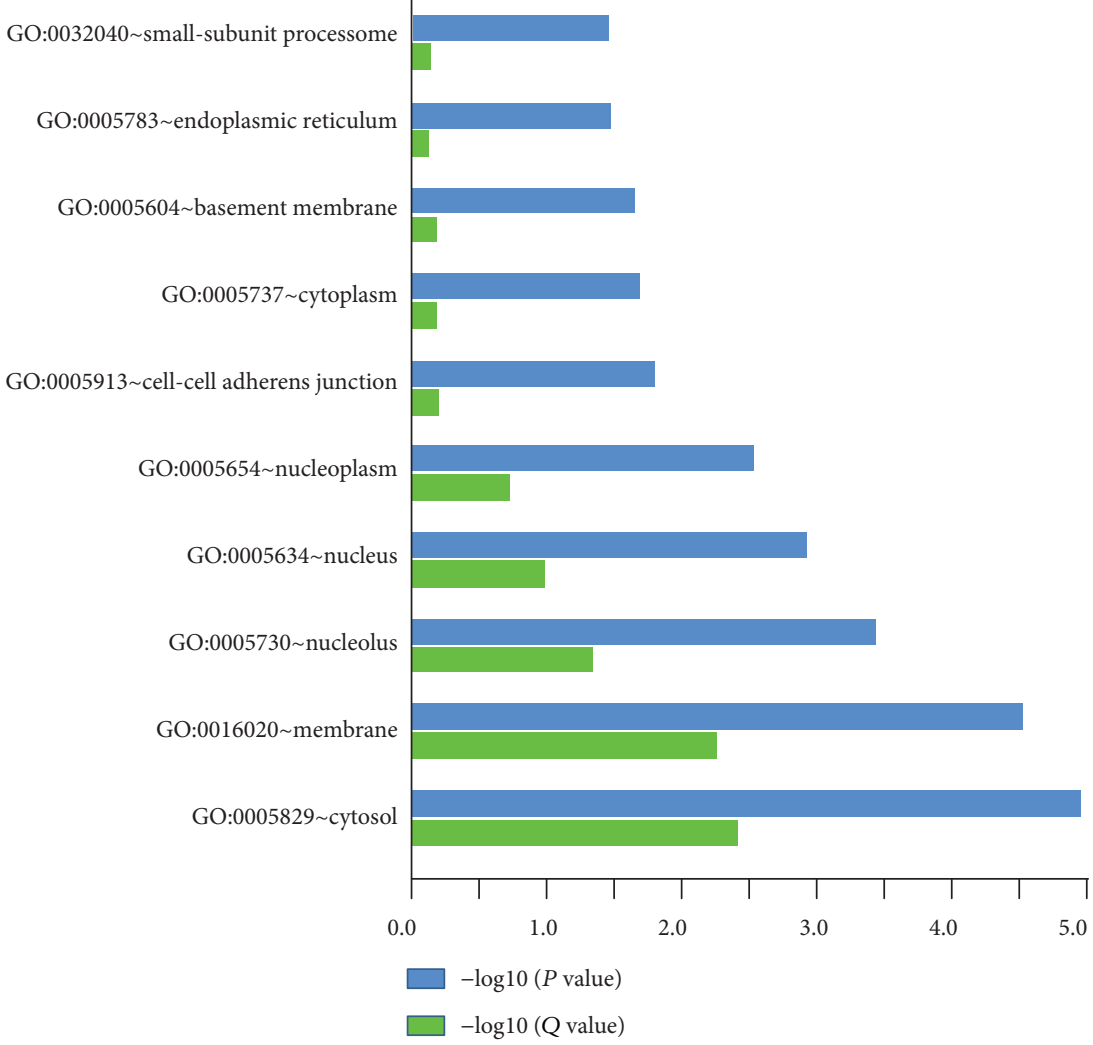

(b)

Figure 3: Continued. 


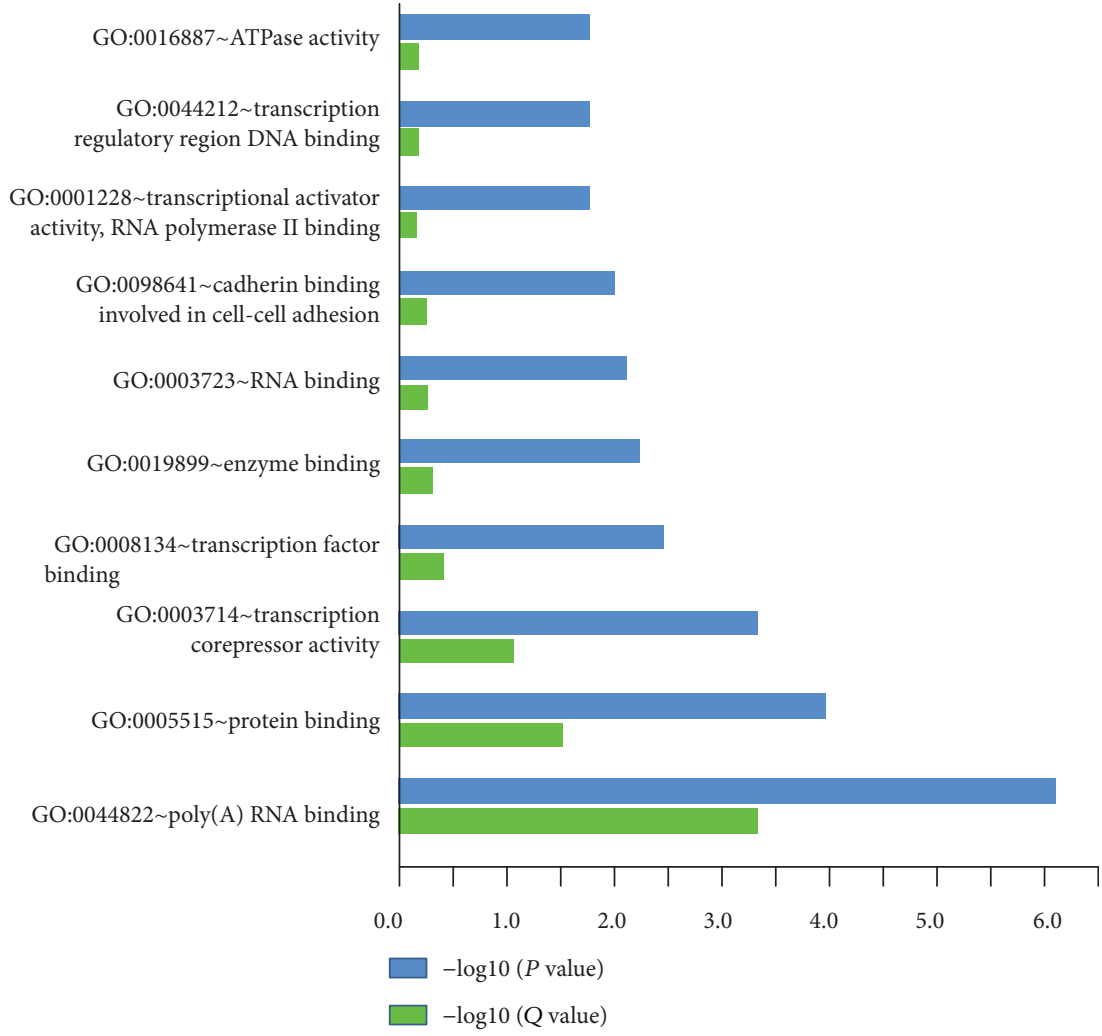

(c)

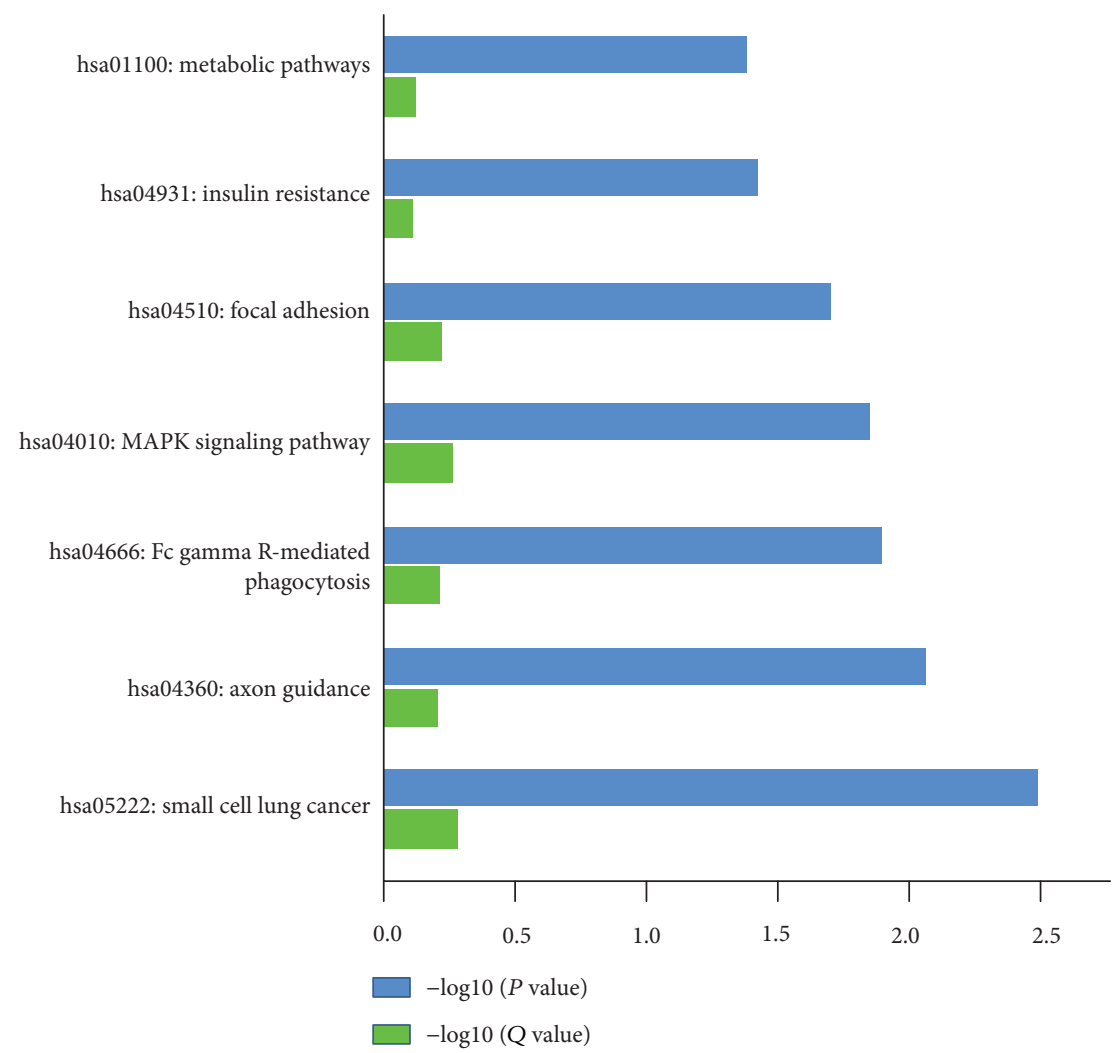

(d)

FIgURe 3: Pathways and summary of GO terms. Enriched GO terms by DAVID (top) and pathway by KEGG (a). The top ten GO enrichment result of biological process (b). The top ten GO enrichment result of cellular component (c). The top ten GO enrichment result of molecular function (d). Enrichment of biological pathway. $P$ values were corrected by the fit linear model. 
TABLE 7: The top 10 cellular components with the highest $P$ values.

\begin{tabular}{lccc}
\hline Go ID & Geneset name & Count & $P$ value \\
\hline GO:0005829 & Cytosol & 102 & $1.09 E-05$ \\
GO:0016020 & Membrane & 73 & $3.07 E-05$ \\
GO:0005730 & Nucleolus & 34 & 0.0004 \\
GO:0005634 & Nucleus & 139 & 0.0012 \\
GO:0005654 & Nucleoplasm & 78 & 0.0029 \\
GO:0005913 & Cell-cell adherens junction & 14 & 0.0158 \\
GO:0005737 & Cytoplasm & 126 & 0.0201 \\
GO:0005604 & Basement membrane & 6 & 0.0227 \\
GO:0005783 & Endoplasmic reticulum & 26 & 0.0330 \\
GO:0032040 & Small-subunit processome & 4 & 0.0341 \\
\hline
\end{tabular}

\section{Discussion}

To date, the CRISPR/Cas9 system is known as a molecular scissor and is widely used in various studies, including cancer research, drug discovery, treatment of mental disease, and applications in plant husbandry [35]. A limitation of this method is that the Cas 9 construct is either permanently integrated or must be later removed with a subsequent reagent delivery and/or clonal selection step to achieve editing without scarring $[36,37]$. In this study, we knocked down Cyr61 via CRISPR/Cas9 plasmid cotransfection with the Cyr61 HDR plasmid. Cyr61 HDR utilizes template DNA (usually the homologous chromosome) to repair DNA in a precise or "error-free" manner [38]. Cyr61 HDR plasmid cotransfected with Cyr61 CRISPR/Cas9 KO plasmid and was designed for repair of the site-specific Cas9-induced DNA cleavage within the Cyr61 gene. During repair, the Cyr61 HDR plasmid incorporates a puromycin resistance gene to enable selection of stable knockout cells and an RFP (red fluorescent protein) gene to visually confirm that the transfection has occurred. Using nonintegrated plasmid vectors that express a puromycin $\mathrm{N}$-acetyltransferase (PAC) gene, whose expression and translation is linked to that of Cas9, we selected for cells based on their early expression levels of Cas9 protein. Edited cells isolated using this method did not contain any detectable off-target mutations and displayed expected functional phenotypes after directed differentiation [38].

Cyr61 is a secreted, extracellular matrix- (ECM-) associated signaling protein of the CCN family [11]. Cyr61 is capable of regulating a broad range of cellular activities, including cell adhesion, migration, proliferation, differentiation, apoptosis, and senescence through interaction with cell surface integrin receptors and heparan sulfate proteoglycans. During embryonic development, Cyr61 is critical for cardiac septal morphogenesis, blood vessel formation in placenta, and vascular integrity. In adulthood, Cyr61 plays important roles in inflammation and tissue repair and is associated with diseases related to chronic inflammation, including rheumatoid arthritis, atherosclerosis, diabetes-related nephropathy and retinopathy, and many different forms of cancers.

lncRNAs are defined as transcripts longer than 200 nucleotides [39]. It is estimated that at least $90 \%$ of RNAs transcribed by the human genome are lncRNAs [40] and was previously considered as "junk gene" or transcriptional "noise" [41]. Although the function of lncRNA remains unclear, in recent years, numerous studies have found that lncRNAs have important biological functions; regulate gene expression via epigenetic regulation, transcriptional regulation, and transcriptional regulation [42]; and participate in embryonic development, cell proliferation, differentiation, apoptosis, and several other biological processes. IncRNAs also play important roles in modulating the innate and adaptive immune responses and immune cell development $[43,44]$. Moreover, emerging evidence suggests that lncRNAs play a decisive role in such autoimmune diseases as systemic lupus erythematosus (SLE), rheumatoid arthritis (RA), type 1 diabetes mellitus (T1DM), multiple sclerosis (MS) [45], autism spectrum disorders [46], tumor biology $[47,48]$, and thoracic and abdominal aortic aneurysms [49]. Future research that deepens our understanding of the biological roles and properties of lncRNAs will enable researchers to harness their potential as valuable diagnostic and therapeutic targets for human diseases.

Unfortunately, until now, Cyr61 functional recovery highlighting its transcriptional networks has not been reported and lncRNAs may play an important regulatory role and possibly mediate Cyr61-involved diseases. To explore the molecular mechanisms involved in Cyr61-induced changes of cell functions, in this study, high-throughput microarray technology designed for genome-wide identification was performed to detect the profiles of aberrantly expressed lncRNAs and mRNAs in downregulated Cyr61 HEK293 cells. A total of 23184 lncRNAs and 28264 mRNAs were normalized. $26 \mathrm{lncRNAs}$ and $212 \mathrm{mRNAs}$ were upregulated, and $74 \mathrm{lncRNAs}$ and 233 mRNAs were downregulated after Cyr61 knockdown. Analysis of the cellular components, molecular functions, biological processes, and regulatory pathways associated with the differentially expressed mRNAs revealed the downstream mechanisms of the Cyr61 gene. The differentially expressed genes were affected for small cell lung cancer, axon guidance, Fc gamma R-mediated phagocytosis, MAPK signaling pathway, focal adhesion, insulin resistance, and metabolic pathways.

Based on pathway analysis, thirteen major mRNAs were found to be involved in the MAPK signaling pathway. The MAPK signaling pathway is essential in regulating numerous cellular processes including inflammation, cell stress response, cell differentiation, cell division, cell proliferation, metabolism, motility, and apoptosis. The role of the MAPK pathway in cancer, immune disorders, and neurodegenerative diseases has been well recognized. The results of gene microarray analysis suggest that downregulation of Cyr61 regulates the cellular function either by regulating the signaling pathway or by regulating the expression of some others genes. This warrants further study.

The mechanisms by which overexpression of Cyr61 increases cancer risk are likely to be multifactorial. Part of the effects seen appears to be due to the direct interaction between Cyr61 protein with the MAPK signaling pathway and p53 as well as an effect on cell apoptosis [7, 50, 51]. An effect mediated by the MAPK signaling pathway is given 
TABle 8: The top 10 molecular functions terms with the highest $P$ values.

\begin{tabular}{lccc}
\hline Go ID & Geneset name & Count & $P$ value \\
\hline GO:0044822 & Poly(A) RNA binding & 51 & $7.30 E-07$ \\
GO:0005515 & Protein binding & 224 & 0.0001 \\
GO:0003714 & Transcription corepressor activity & 14 & 0.0004 \\
GO:0008134 & Transcription factor binding & 15 & 0.0033 \\
GO:0019899 & Enzyme binding & 16 & 0.0055 \\
GO:0003723 & RNA binding & 22 & 0.0074 \\
GO:0098641 & Cadherin binding involved in cell-cell adhesion & 14 & 0.0098 \\
GO:0001228 & Transcriptional activator activity, RNA polymerase II transcription & 7 & 0.0162 \\
GO:0044212 & regulatory region sequence-specific binding & 11 & 10 \\
GO:0016887 & Transcription regulatory region DNA binding & 0.0165 \\
\hline
\end{tabular}

TABle 9: The top 7 molecular signaling pathways for KEGG pathway analysis with the highest $P$ values.

\begin{tabular}{lccc}
\hline Pathway ID & Definition & Count & $P$ value \\
\hline Hsa05222 & Small cell lung cancer & 8 & 0.0032 \\
Hsa04360 & Axon guidance & 9 & 0.0084 \\
Hsa04666 & Fc gamma R-mediated phagocytosis & 7 & 0.0122 \\
Hsa04010 & MAPK signaling pathway & 13 & 0.0136 \\
Hsa04510 & Focal adhesion & 11 & 0.0194 \\
Hsa04931 & Insulin resistance & 7 & 0.0369 \\
Hsa01100 & Metabolic pathways & 38 & 0.0415 \\
\hline
\end{tabular}

additional support by our transcriptome analysis. In addition, based on our findings, Cyr61 may also contribute to malignant transformation indirectly through its effects on other genes, which were found to be differentially expressed in this study and have been implicated in malignant transformation. These genes including SERPINF1, CRABP2, and TXNIP [52-54]. REG3G, ACAT2, and ATF5 have also been found in various cancers [55-57]. Several of the differentially expressed lncRNAs we show in Table 4 have also been found to be associated with cancer development, including $\mathrm{CH} 17-$ 360D5.2, SOX2-OT, and HOXA11 [58-60]. These findings suggest that the effect of Cyr61 on malignant transformation is multifactorial and not simply due to a direct interaction with the MAPK signaling pathway.

Additionally, we also found that the differently expressed genes are involved in other pathophysiological processes unrelated to cancer. For mRNAs, for example, PRR21 may be involved in stress responses that are related to phosphorylation of mitochondrial proteins [61]. Mutation of PIGW is associated with West syndrome and hyperphosphatasia with mental retardation syndrome [62]. Rbm19 plays an important role in the development of intestinal epithelium cells [63]. Thus, the genetic changes associated with Cyr61 may be involved in the occurrence and development of many diseases.

GO analysis predicted that up- and downregulated mRNAs were associated with several biological processes, cellular components, and molecular functions in HEK 293 cells with downregulated Cyr61. The GO annotation indicated that these gene products were attributed to the progress of a variety of biochemical reactions, such as cell-cell adhesion, negative regulation of cell migration, protein binding, mRNA processing, cell-cell adherens junction and poly $(\mathrm{A})$ RNA binding. Cell adhesion and migration are the central processes in the development and maintenance of multicellular organisms. Tissue formation during embryonic development, wound healing, and immune responses all require the orchestrated movement of cells in particular directions to specific locations. Errors during these processes may have serious consequences, including intellectual disability, vascular disease, tumor formation, and metastasis [64].

It has been reported that Cyr61 mediates numerous cellular activities, including cell migration, adhesion, apoptosis, and proliferation [65]. As a cell adhesion protein, through binding to particular integrins, Cyr61 associates with the adhesion activities of endothelial cells, fibroblast cells, smooth muscle cells, and monocytes [66-69]. Numerous studies have shown that Cyr61 is involved in cell migration, including smooth muscle cell migration, fibroblast-like synoviocyte invasion, and cancerous adenocarcinoma [70-72], and is seen as a promising therapeutic target. Nevertheless, the mechanism by which Cyr61 participates in cell adhesion and migration is still not completely clear because both cell adhesion and migration are complex biological processes. Our research shows that a large number of abnormal genes are involved in cell adhesion and migration. It is anticipated that this can provide additional research directions for Cyr61-involved diseases. Subsequently, exploration of the correlations between abnormal genes and signal pathways is critical for developing new biomarkers for the early diagnosis and therapeutic surveillance of Cyr61-involved diseases.

We also studied the correlation between Ang II and Cyr61 in HEK293 cells. It is well recognized that the RAS is an important hormonal system in humans and exists not only as a circulating and paracrine but also as an intracrine system [20, 73]. There have been many well-documented pathophysiologic functions of the intrarenal RAS. Increasing 


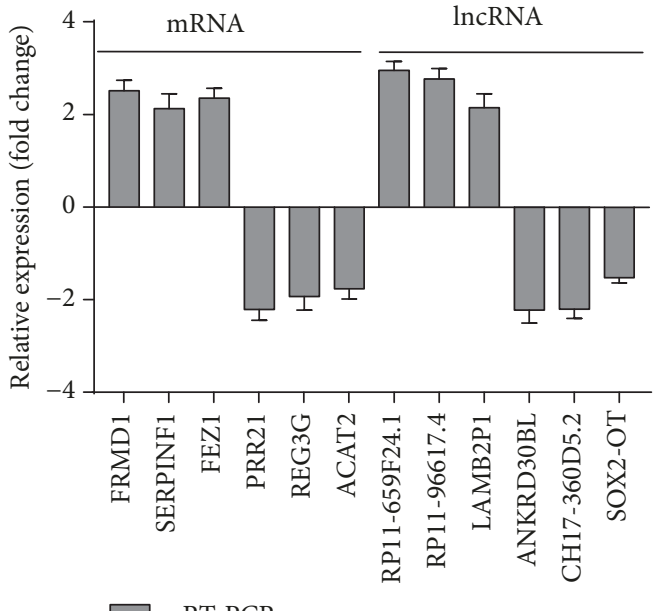

qRT-PCR

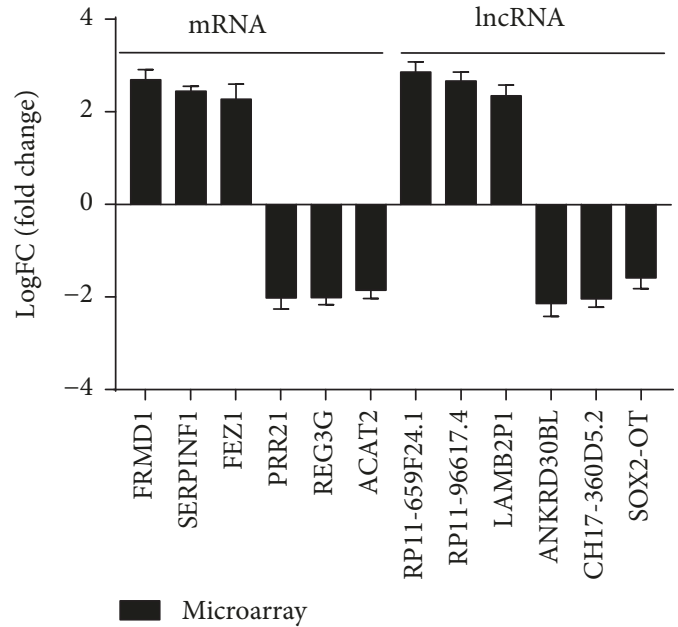

(b)

Figure 4: Validation of mRNA and lncRNA expression by RT-qPCR. The RT-qPCR validation of six mRNAs and lncRNAs. The results showed that the expression levels of the mRNAs FRMD1, SERPINF1, and FEZ1 and lncRNAs RP11-659F24.1, RP11-966I7.4, and LAMB2P1 were upregulated compared with the controls, and the expression levels of the mRNAs PRR21, REG3G, and ACAT2 and IncRNAs ANKRD30BL, CH17-360D5.2, and SOX2-OT were downregulated compared with the controls (a). The results of RT-qPCR were similar to those obtained from the microarray analysis (b).

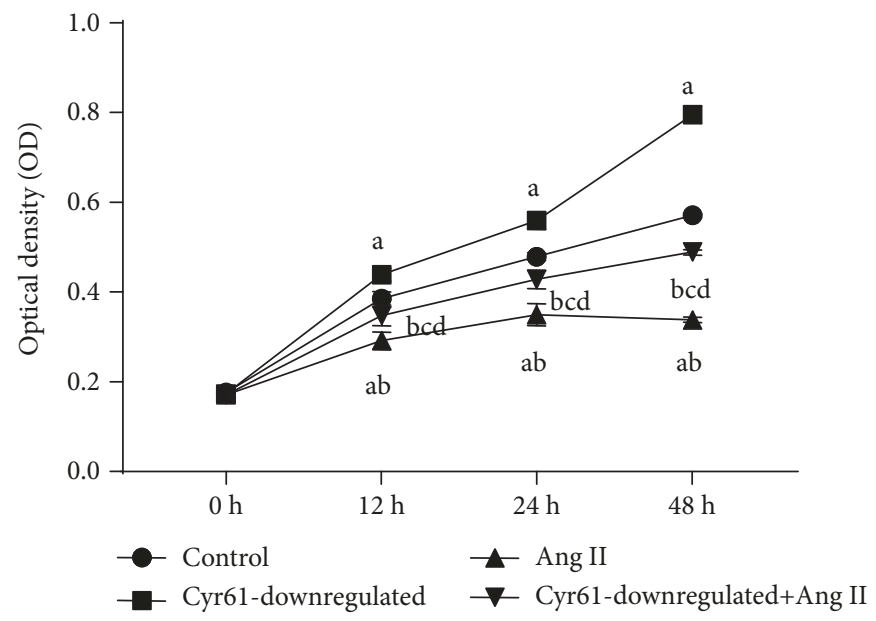

FIgURE 5: Effects of Cyr61 on Ang II-induced proliferation in HEK293T cells. To investigate the effects of Cyr61 on Ang II-induced proliferation in HEK293T cells, the HEK293T cells were treated with Ang II after knocking down the Cyr61 gene. Proliferation was estimated using Cell Counting Kit- 8 assay. ${ }^{a} P<0.05$ compared with controls, ${ }^{b} P<0.05$ compared with Cyr61 knockdown, ${ }^{c} P<0.05$ compared with Ang II without Cyr61 knockdown, and ${ }^{\mathrm{d}} P>0.05$ compared with controls. The data represent means \pm SD from 3 independent experiments.

lines of evidence have indicated the RAS was involved in a variety of diseases including tumors, depression, glaucomatous, cardiovascular diseases, kidney diseases, and Parkinson's disease [74-78]. The pathological processes include fibrosis, inflammation, and immune responses, and it also induces inflammation, renal cell growth, mitogenesis, apoptosis, migration, and differentiation [79], and the adverse effects of the RAS on these diseases are due mainly to increased levels of Ang II, which exerts its effects through the classical ACE-AngII-AT1R axis [80].
In this study, we knocked down the Cyr61 gene of HEK293T cells by using the Cyr61 CRISPR/Cas9 KO plasmid and Cyr61 HDR transfection plasmid and then treated the HEK293T cells with Ang II. The cell functions of these HEK293T cells were then observed including the cell cycle, cell proliferation, and cell apoptosis. Our results showed that the expression of Cyr61 protein was significantly decreased after knocking down the Cyr61 gene by Cyr61 CRISPR/Cas9 KO plasmid and HDR transfection plasmid in HEK293T cells compared with the controls, and knockdown of the 


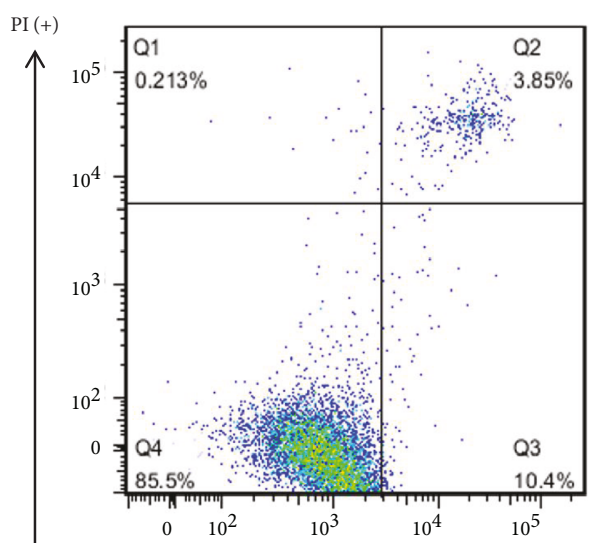

(A)

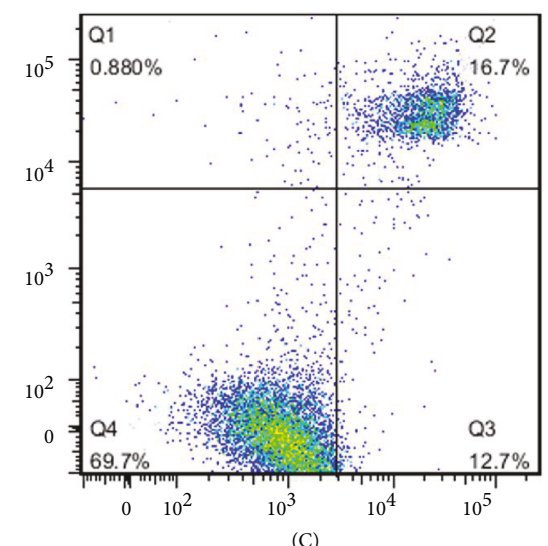

(C)
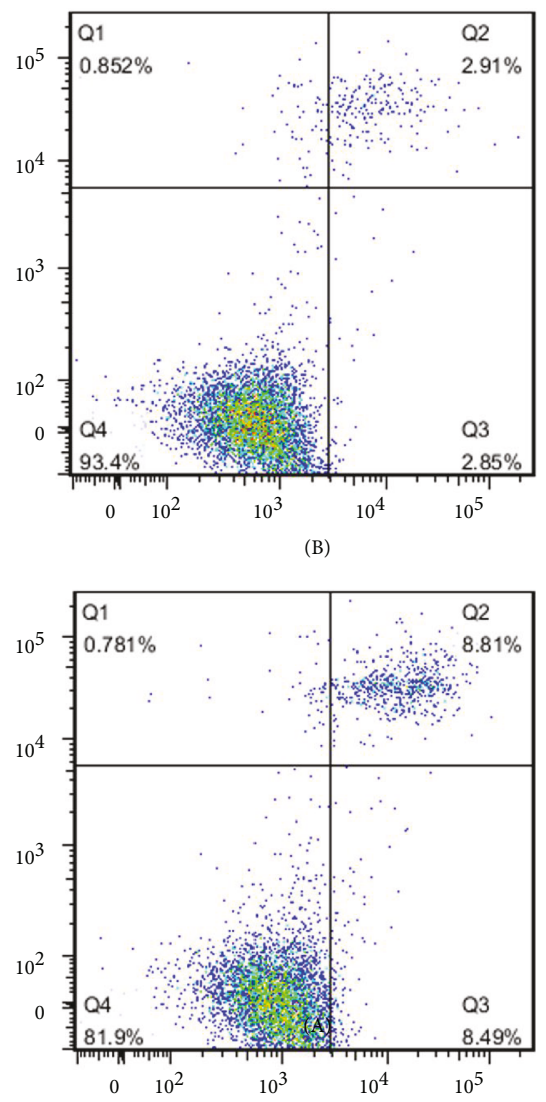

(D)

A: control, B: Cyr61-downregulated, C: Ang II

D: Cyr61-downregulated+Ang II

(a)

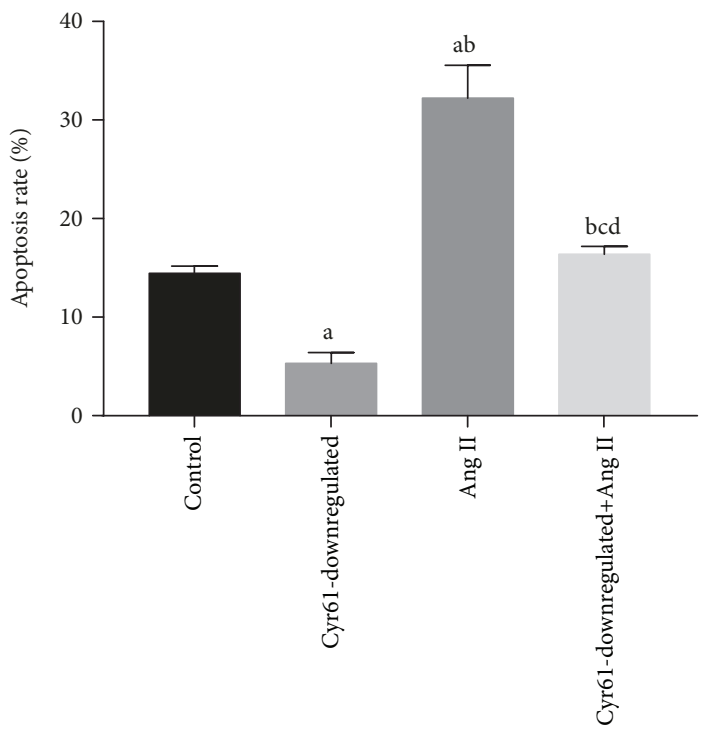

(b)

FIGURE 6: Effects of Cyr61 on Ang II-induced apoptosis in HEK293T cells. To investigate the effects of Cyr61 on Ang II-induced apoptosis on HEK293T cells (a). the HEK293T cells were treated with Ang II after knocking down the Cyr61 gene for $48 \mathrm{~h}$, and apoptosis was measured by Annexin V-FITC/PI staining and flow cytometry. (b). The ratio of apoptotic cells to the total number of cells. The number of apoptotic cells equals the sum of the cells in the Q2 (early-stage cell apoptosis rate) and Q3 (late-stage cell apoptosis rate). ${ }^{\mathrm{a}} \mathrm{P}<0.05$ compared with controls, ${ }^{\mathrm{b}} P<0.05$ compared with Cyr61 knockdown, ${ }^{\mathrm{c}} P<0.05$ compared with Ang II without Cyr61 knockdown, and ${ }^{\mathrm{d}} P>0.05$ compared with controls. The data represent means \pm SD from 3 independent experiments. 


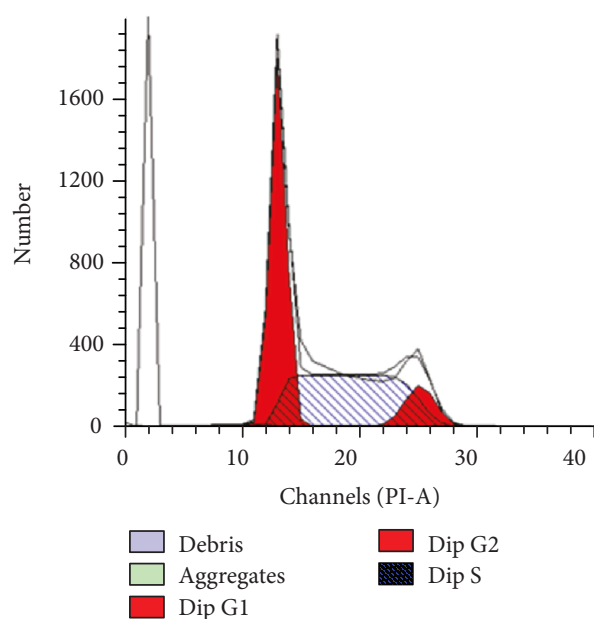

(A)

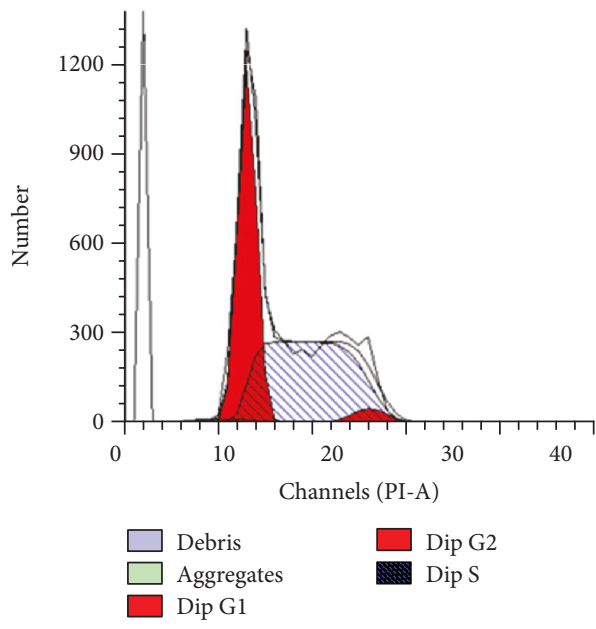

(C)

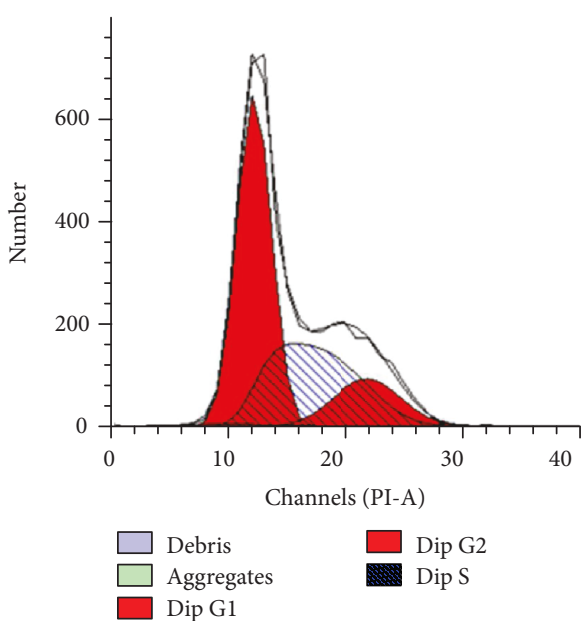

(B)

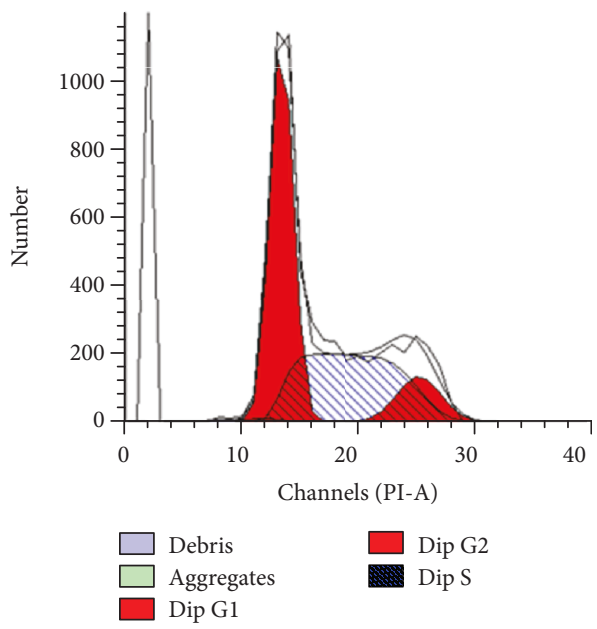

(D)

A: Control B: Cyr61-downregulated, C: Ang II, D: Cyr61-downregulated+Ang II

(a)

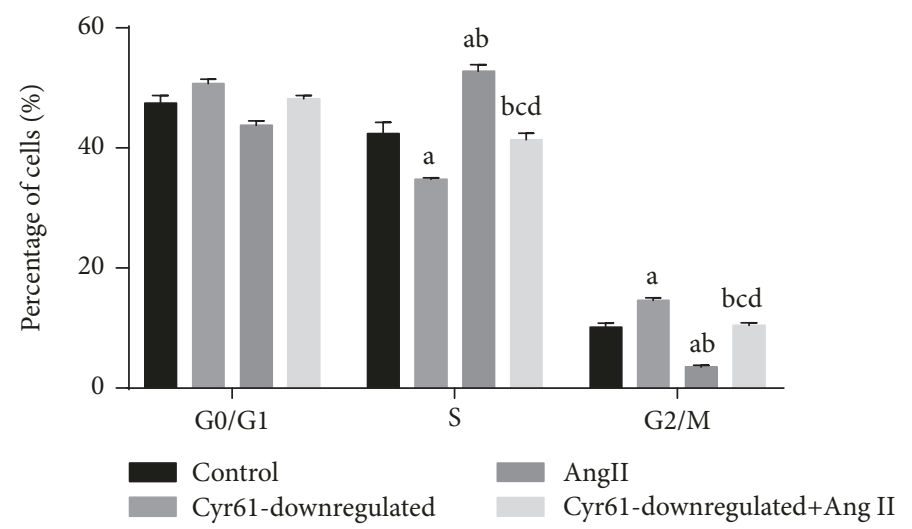

(b)

FIGURE 7: Effects of Cyr61 on Ang II-induced cell cycle progression in HEK293T cells. To investigate the effects of Cyr61 on Ang II-induced cycle progression in HEK293T cells, the HEK293T cells were treated with Ang II after knocking down the Cyr61 gene for 48 h. Cells were stained with PI and collected to be examined on a flow cytometer. ${ }^{a} P<0.05$ compared with controls, ${ }^{b} P<0.05$ compared with Cyr61 knockdown, ${ }^{c} P<0.05$ compared with Ang II without Cyr61 knockdown, and ${ }^{\mathrm{d}} P>0.05$ compared with controls. The data represent means \pm SD from 3 independent experiments. 


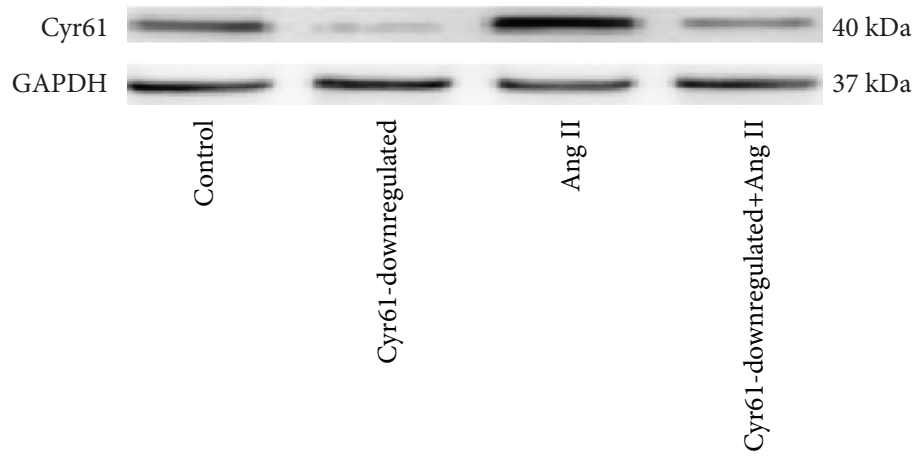

(a)

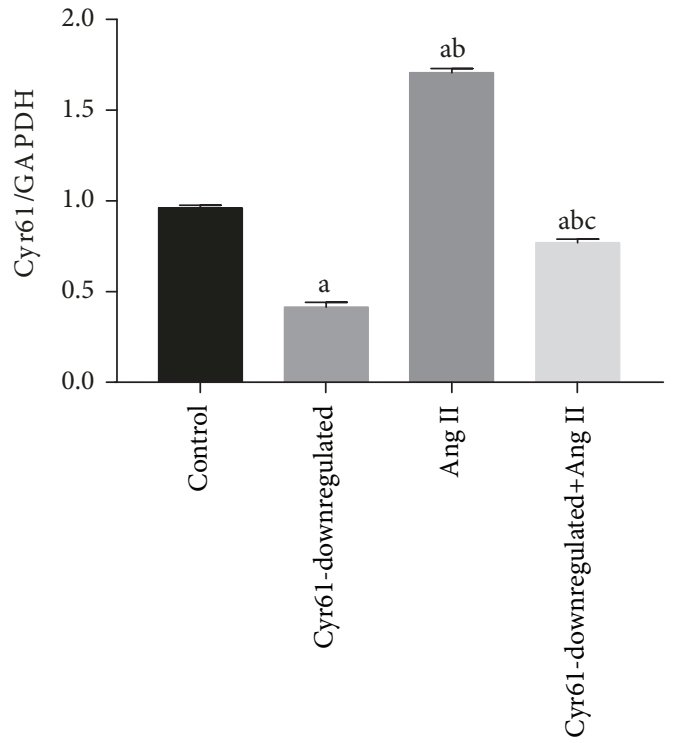

(b)

Figure 8: The expression of Cyr61 protein treated with Ang II. To investigate whether Ang II induces Cyr61 expression in 293 cells, we incubated HEK293T cells for 48 hours in DMEM with or without Ang II. (a). Western blots were performed to detect the expression levels of GAPDH and Cyr61 protein in HEK293T cells. (b) Quantitative analysis of Cyr61 protein. GAPDH was used as internal control. Statistical analysis was performed using one-way ANOVA. ${ }^{\mathrm{a}} P<0.05$ compared with controls, ${ }^{\mathrm{b}} \mathrm{P}<0.05$ compared with Cyr61 knockdown, and ${ }^{\mathrm{c}} P<0.05$ compared with Ang II without Cyr61 knockdown. The data represent means \pm SD from 3 independent experiments.

Cry61 gene can promote cell proliferation and inhibit apoptosis significantly. These results may be related to promoting the cell cycle. In this study, cell-cycle distribution analysis showed significantly decreased cell populations in the $S$ phase and increased cell populations in the G2/M phase when the Cyr61 gene was knocked down. Our results are similar to those of some previous studies. For example, Xiong et al. reported that Ang II significantly inhibited cell survival, induced cell cycle arrest, and enhanced cell apoptosis in HK2 cells [81].

Interestingly, the HEK293T cells with knockdown of the Cyr61 gene treated with Ang II have a higher cell proliferation rate and $\mathrm{G} 2 / \mathrm{M}$ phase and lower apoptosis rate than the Ang II-added group of cells. We further examined the expression of Cyr61 after treating with Ang II. Consistent with the findings of Hilfiker et al. [82], we found that Cyr61 transcript levels were elevated in HEK293T cells exposed to Ang II compared with controls. Furthermore, the Cyr61 protein transcript levels of HEK293T cells with the Cyr61 gene knocked down were elevated significantly after treating with Ang II compared to cells without Ang II treatment. In contrast, the proliferation and apoptosis of Cyr61-downregulated + Ang II cells were not different to control cells, although the Cyr61 level of downregulated + Ang II cells was lower than that of the controls was. This probably means that Cyr61 is not the only factor which is involved in Ang II-induced apoptosis and antiproliferation of these cells and suggests that some others factors and signaling pathways, such as AT2 receptor (angiotensin type 2 receptor), p38 signaling pathway, and IGF-IR-PI3K-Akt signaling are also involved in these processes [83-85]. Accordingly, further research into the mechanisms of Ang
II-induced apoptosis and antiproliferation is needed. Taken together, downregulation of Cyr61 is of benefit to the cell cycle and is antiapoptotic, and Ang II induces HEK293T cell injury, at least in part, by upregulating the expression of Cyr61 protein.

There are some limitations in our study. The cells transfected with the vector that expresses puromycin $\mathrm{N}$ transferase (PAC) were not involved, and the unspecific effects of PAC on HEK293T cells were not observed in the present study. The puromycin selection may enhance Cas- 9 expression in the transfected cells so unspecific effects by Cas- 9 cannot be ruled out. lncRNAs can play their biological functions by interacting with mRNAs. The biological meanings of differentially expressed lncRNAs will be more convinced if the lncRNAs and mRNA coexpression network can be performed between the validated lncRNAs and their related mRNA based on the correlation analysis.

\section{Conclusions}

Cyr61 is mutated in a variety of diseases. We report for the first time that many lncRNAs and mRNAs are significantly upregulated or downregulated and that specific metabolic pathways may play important roles in the downstream events of the Cyr61 gene. The significantly altered lncRNAs and mRNAs levels may be related to many pathological processes. It appears that Cyr61 is involved in Ang IIinduced injury in HEK293T cells. It is envisaged that studies of the functional mechanisms of these differentially expressed lncRNAs and mRNAs as well as exploration of the pathways involved will provide novel targets for Cyr61-involved diseases. 


\section{Data Availability}

The data used to support the findings of this study are available from the corresponding author upon request.

\section{Ethical Approval}

The study was approved by the Committee of Animal Care and Use of Youjiang Medical University for Nationalities, and all procedures were performed according to the National Institute of Health Guidelines.

\section{Conflicts of Interest}

The authors have no conflicts of interest to declare.

\section{Authors' Contributions}

Junjie Wang, Dongdong Fu, and Soulixay Senouthai carried out the experimental work and conceived of the study and participated in its design and coordination. Yan Jiang and Rentong $\mathrm{Hu}$ participated in the design of the study and together with Yanwu You performed the statistical analysis. Junjie Wang and Yanwu You drafted the manuscript. All authors read and approved the final manuscript. Junjie Wang, Dongdong Fu, and Soulixay Senouthai are co-first authors.

\section{Acknowledgments}

This study was supported by grants from the National Natural Science Foundation of China (No. 81560271), the Key Project of Scientific Research of the Guangxi Colleges and Universities (No. KY2015ZD092), and the Program of Natural Science Foundation of Guangxi (Nos. 2014GXNSFAA118253 and 2017GXNSFDA198005). The authors would like to thank Dr. Dev Sooranna, Imperial College London, for editing the manuscript.

\section{References}

[1] Y. Emre and B. A. Imhof, "Matricellular protein CCN1/CYR61: a new player in inflammation and leukocyte trafficking," Seminars in Immunopathology, vol. 36, no. 2, pp. 253-259, 2014.

[2] L. F. Lau, "Cell surface receptors for CCN proteins," Journal of Cell Communication and Signaling, vol. 10, no. 2, pp. 121-127, 2016.

[3] T. Xu, Y. H. He, M. Q. Wang et al., "Therapeutic potential of cysteine-rich protein 61 in rheumatoid arthritis," Gene, vol. 592, no. 1, pp. 179-185, 2016.

[4] B. Lebas, J. Galley, E. Renaud-Gabardos et al., "Therapeutic benefits and adverse effects of combined proangiogenic gene therapy in mouse critical leg ischemia," Annals of Vascular Surgery, vol. 40, no. 11, pp. 252-261, 2017.

[5] J. Zhang, G. Wu, and H. Dai, "The matricellular protein CCN1 regulates TNF- $\alpha$ induced vascular endothelial cell apoptosis," Cell Biology International, vol. 40, no. 1, pp. 1-6, 2016.

[6] Y. Liu, F. Zhang, Z. Zhang et al., "High expression levels of Cyr61 and VEGF are associated with poor prognosis in osteosarcoma," Pathology, Research and Practice, vol. 213, no. 8, pp. 895-899, 2017.

[7] K. B. Lee, H. J. Byun, S. H. Park, C. Y. Park, S. H. Lee, and S. B. Rho, "CYR61 controls p53 and NF- $\kappa$ B expression through $\mathrm{PI} 3 \mathrm{~K} / \mathrm{Akt} / \mathrm{mTOR}$ pathways in carboplatin-induced ovarian cancer cells," Cancer Letters, vol. 315, no. 1, pp. 86-95, 2012.

[8] M. T. Lin, C. C. Chang, S. T. Chen et al., "Cyr61 expression confers resistance to apoptosis in breast cancer MCF-7 cells by a mechanism of NF- $\kappa$ B-dependent XIAP up-regulation," Journal of Biological Chemistry, vol. 279, no. 23, pp. 2401524023, 2004.

[9] X. Tong, J. O'Kelly, D. Xie et al., "Cyr61 suppresses the growth of non-small-cell lung cancer cells via the $\beta$-catenin-c-mycp53 pathway," Oncogene, vol. 23, no. 28, pp. 4847-4855, 2004.

[10] C. P. Pilarsky, U. Schmidt, C. Eissrich et al., "Expression of the extracellular matrix signaling molecule Cyr61 is downregulated in prostate cancer," Prostate, vol. 36, no. 2, pp. 85-91, 1998.

[11] L. F. Lau, "CCN1/CYR61: the very model of a modern matricellular protein," Cellular and Molecular Life Sciences, vol. 68, no. 19, pp. 3149-3163, 2011.

[12] J. M. Perkel, "Visiting "noncodarnia"," BioTechniques, vol. 54, no. 6, 2013

[13] L. J. Li, R. X. Leng, Y. G. Fan, H. F. Pan, and D. Q. Ye, “Translation of noncoding RNAs: focus on lncRNAs, pri-miRNAs, and circRNAs," Experimental Cell Research, vol. 361, no. 1, pp. 1-8, 2017.

[14] P. Kapranov, J. Cheng, S. Dike et al., "RNA maps reveal new RNA classes and a possible function for pervasive transcription," Science, vol. 316, no. 5830, pp. 1484-1488, 2007.

[15] K. J. Yin, M. Hamblin, and Y. E. Chen, "Non-coding RNAs in cerebral endothelial pathophysiology: emerging roles in stroke," Neurochemistry International, vol. 77, pp. 9-16, 2014.

[16] F. Khan, H. Chai, I. Ajmera, C. Hodgman, S. Mayes, and C. Lu, "A transcriptomic comparison of two Bambara groundnut landraces under dehydration stress," Genes, vol. 8, no. 4, p. 121, 2017.

[17] C. Hanjin, L. Tao, L. Pengfei et al., “Altered long noncoding RNA and messenger RNA expression in experimental intracerebral hemorrhage - a preliminary study," Cellular Physiology and Biochemistry, vol. 45, no. 3, pp. 1284-1301, 2018.

[18] A. Zhou, H. Ma, E. Liu et al., "Transcriptome sequencing of Dianthus spiculifolius and analysis of the genes involved in responses to combined cold and drought stress," International Journal of Molecular Sciences, vol. 18, no. 4, p. 849, 2017.

[19] H. Quan, M. Liang, N. Li et al., "LncRNA-AK131850 sponges MiR-93-5p in newborn and mature osteoclasts to enhance the secretion of vascular endothelial growth factor a promoting Vasculogenesis of endothelial progenitor cells," Cellular Physiology and Biochemistry, vol. 46, no. 1, pp. 401417, 2018.

[20] R. Kumar, C. M. Thomas, Q. C. Yong, W. Chen, and K. M. Baker, "The intracrine renin-angiotensin system," Clinical Science (London, England), vol. 123, no. 5, pp. 273-284, 2012.

[21] P. Sobczuk, C. Szczylik, C. Porta, and A. Czarnecka, "Renin angiotensin system deregulation as renal cancer risk factor (Review)," Oncology Letters, vol. 14, no. 5, pp. 5059-5068, 2017.

[22] F. Fyhrquist and O. Saijonmaa, "Renin-angiotensin system revisited," Journal of Internal Medicine, vol. 264, no. 3, pp. 224-236, 2008. 
[23] M. A. Sparks, S. D. Crowley, S. B. Gurley, M. Mirotsou, and T. M. Coffman, "Classical renin-angiotensin system in kidney physiology," Comprehensive Physiology, vol. 4, no. 3, pp. 12011228, 2014.

[24] R. Igic and R. Skrbic, "The renin-angiotensin system and its blockers," Srpski Arhiv za Celokupno Lekarstvo, vol. 142, no. 11-12, pp. 756-763, 2014.

[25] R. A. S. Santos, A. J. Ferreira, T. Verano-Braga, and M. Bader, "Angiotensin-converting enzyme 2, angiotensin(1-7) and Mas: new players of the renin-angiotensin system," Journal of Endocrinology, vol. 216, no. 2, pp. R1R17, 2013.

[26] Z. Xu, W. Li, J. Han et al., "Angiotensin II induces kidney inflammatory injury and fibrosis through binding to myeloid differentiation protein-2 (MD2)," Scientific Reports, vol. 7, no. 1, article 44911, 2017.

[27] A. Miyajima, T. Kosaka, E. Kikuchi, and M. Oya, "Reninangiotensin system blockade: Its contribution and controversy," International Journal of Urology, vol. 22, no. 8, pp. 721-730, 2015.

[28] M. A. Saleh, A. E. Norlander, and M. S. Madhur, "Inhibition of interleukin-17A, but not interleukin-17F, signaling lowers blood pressure, and reduces end-organ inflammation in angiotensin II-induced hypertension," JACC: Basic to Translational Science, vol. 1, no. 7, pp. 606-616, 2016.

[29] Y. Zhang, H. Yan, G. C. Guang, and Z. R. Deng, "Overexpressed connective tissue growth factor in cardiomyocytes attenuates left ventricular remodeling induced by angiotensin II perfusion," Clinical and Experimental Hypertension, vol. 39, no. 2, pp. 168-174, 2017.

[30] D. E. Richard, V. Vouret-Craviari, and J. Pouyssegur, “Angiogenesis and G-protein-coupled receptors: signals that bridge the gap," Oncogene, vol. 20, no. 13, pp. 1556-1562, 2001.

[31] D. Weiss, D. Sorescu, and W. R. Taylor, "Angiotensin II and atherosclerosis," The American Journal of Cardiology, vol. 87, no. 8, Supplement 1, pp. 25-32, 2001.

[32] J. Chen, M. Lin, A. Hrabovsky et al., "ZNF804A transcriptional networks in differentiating neurons derived from induced pluripotent stem cells of human origin," PLoS One, vol. 10, no. 4, article e0124597, 2015.

[33] P. R. Burkett, G. Meyer zu Horste, and V. K. Kuchroo, "Pouring fuel on the fire: Th17 cells, the environment, and autoimmunity," The Journal of Clinical Investigation, vol. 125, no. 6 , pp. 2211-2219, 2015.

[34] Q. Yang, Y. Ma, Y. Liu et al., “Angiotensin II down-regulates nephrin-Akt signaling and induces podocyte injury: role of c-Abl," Molecular Biology of the Cell, vol. 27, no. 1, pp. 197208, 2016.

[35] J. Yang, X. Meng, J. Pan et al., "CRISPR/Cas9-mediated noncoding RNA editing in human cancers," RNA Biology, vol. 15, no. 1, pp. 35-43, 2018.

[36] J. Cao, L. Wu, S. M. Zhang et al., "An easy and efficient inducible CRISPR/Cas9 platform with improved specificity for multiple gene targeting," Nucleic Acids Research, vol. 44, no. 19, article e149, 2016.

[37] G. Wang, L. Yang, D. Grishin et al., "Efficient, footprint-free human iPSC genome editing by consolidation of Cas9/CRISPR and piggyBac technologies," Nature Protocols, vol. 12, no. 1, pp. 88-103, 2017.

[38] B. Steyer, Q. Bu, E. Cory et al., "Scarless genome editing of human pluripotent stem cells via transient puromycin selection," Stem Cell Reports, vol. 10, no. 2, pp. 642-654, 2018.

[39] M. S. Kowalczyk, D. R. Higgs, and T. R. Gingeras, "Molecular biology: RNA discrimination," Nature, vol. 482, no. 7385, pp. 310-311, 2012.

[40] S. Djebali, C. A. Davis, A. Merkel et al., "Landscape of transcription in human cells," Nature, vol. 489, no. 7414, pp. 101-108, 2012.

[41] A. F. Palazzo and E. S. Lee, "Non-coding RNA: what is functional and what is junk?," Frontiers in Genetics, vol. 6, p. 2, 2015.

[42] D. P. Caley, R. C. Pink, D. Trujillano, and D. R. F. Carter, "Long noncoding RNAs, chromatin, and development," TheScientificWorldJournal, vol. 10, pp. 90-102, 2010.

[43] T. M. Aune and C. F. Spurlock III, "Long non-coding RNAs in innate and adaptive immunity," Virus Research, vol. 212, pp. 146-160, 2016.

[44] Z. Wang and Y. Zheng, "IncRNAs regulate innate immune responses and their roles in macrophage polarization," Mediators of Inflammation, vol. 2018, Article ID 8050956, 8 pages, 2018.

[45] G. C. Wu, H. F. Pan, R. X. Leng et al., "Emerging role of long noncoding RNAs in autoimmune diseases," Autoimmunity Reviews, vol. 14, no. 9, pp. 798-805, 2015.

[46] J. Tang, Y. Yu, and W. Yang, "Long noncoding RNA and its contribution to autism spectrum disorders," CNS Neuroscience \& Therapeutics, vol. 23, no. 8, pp. 645-656, 2017.

[47] H. Li, X. Yuan, D. Yan et al., "Long non-coding RNA MALAT1 decreases the sensitivity of resistant glioblastoma cell lines to temozolomide," Cellular Physiology and Biochemistry, vol. 42, no. 3, pp. 1192-1201, 2017.

[48] R. Yang, P. Li, G. Zhang, C. Lu, H. Wang, and G. Zhao, "Long non-coding RNA XLOC_008466 functions as an oncogene in human non-small cell lung cancer by targeting miR-874," Cellular Physiology and Biochemistry, vol. 42, no. 1, pp. 126-136, 2017.

[49] Y. Li and L. Maegdefessel, "Non-coding RNA contribution to thoracic and abdominal aortic aneurysm disease development and progression," Frontiers in Physiology, vol. 8, p. $429,2017$.

[50] J. A. Menendez, L. Vellon, I. Mehmi, P. K. Teng, D. W. Griggs, and R. Lupu, "A novel CYR61-triggered 'CYR61- $\alpha_{\mathrm{v}} \beta_{3}$ integrin loop' regulates breast cancer cell survival and chemosensitivity through activation of ERK1/ERK2 MAPK signaling pathway," Oncogene, vol. 24, no. 5, pp. 761-779, 2005.

[51] R. Yang, Y. Chen, and D. Chen, "Biological functions and role of CCN1/Cyr61 in embryogenesis and tumorigenesis in the female reproductive system (review)," Molecular Medicine Reports, vol. 17, no. 1, pp. 3-10, 2018.

[52] B. Xu, J. Li, X. Liu, C. Li, and X. Chang, "TXNDC5 is a cervical tumor susceptibility gene that stimulates cell migration, vasculogenic mimicry and angiogenesis by down-regulating SERPINF1 and TRAF1 expression," Oncotarget, vol. 8, no. 53, pp. 91009-91024, 2017.

[53] R. Z. Liu, S. Li, E. Garcia et al., “Association between cytoplasmic CRABP2, altered retinoic acid signaling, and poor prognosis in glioblastoma," Glia, vol. 64, no. 6, pp. 963976, 2016.

[54] Y. Zhou, J. Zhou, X. Lu, T. Z. Tan, and W. J. Chng, "BET bromodomain inhibition promotes De-repression of TXNIP and activation of ASK1-MAPK pathway in acute myeloid leukemia," BMC Cancer, vol. 18, no. 1, p. 731, 2018. 
[55] X. Liu, Z. Zhou, Q. Cheng et al., “Acceleration of pancreatic tumorigenesis under immunosuppressive microenvironment induced by Reg3g overexpression," Cell Death \& Disease, vol. 8, no. 9, p. e3033, 2017.

[56] Y. Huang, Q. Jin, M. Su et al., "Leptin promotes the migration and invasion of breast cancer cells by upregulating ACAT2," Cellular Oncology (Dordrecht), vol. 40, no. 6, pp. 537-547, 2017.

[57] J. M. Angelastro, "Targeting ATF5 in cancer," Trends in Cancer, vol. 3, no. 7, pp. 471-474, 2017.

[58] R. Oka, K. I. Nakashiro, H. Goda, K. Iwamoto, N. Tokuzen, and H. Hamakawa, "Annexin A8 is a novel molecular marker for detecting lymph node metastasis in oral squamous cell carcinoma," Oncotarget, vol. 7, no. 4, pp. 4882-4889, 2016.

[59] Z. Wang, M. Tan, G. Chen, Z. Li, and X. Lu, "LncRNA SOX2OT is a novel prognostic biomarker for osteosarcoma patients and regulates osteosarcoma cells proliferation and motility through modulating SOX2," IUBMB Life, vol. 69, no. 11, pp. 867-876, 2017.

[60] F. Q. Yang, J. Q. Zhang, J. J. Jin et al., "HOXA11-AS promotes the growth and invasion of renal cancer by sponging miR146b-5p to upregulate MMP16 expression," Journal of Cellular Physiology, vol. 233, no. 12, pp. 9611-9619, 2018.

[61] Y. Kanamaru, S. Sekine, H. Ichijo, and K. Takeda, "The phosphorylation-dependent regulation of mitochondrial proteins in stress responses," Journal of Signal Transduction, vol. 2012, Article ID 931215, 12 pages, 2012.

[62] T. Chiyonobu, N. Inoue, M. Morimoto, T. Kinoshita, and Y. Murakami, "Glycosylphosphatidylinositol (GPI) anchor deficiency caused by mutations in PIGW is associated with West syndrome and hyperphosphatasia with mental retardation syndrome," Journal of Medical Genetics, vol. 51, no. 3, pp. 203-207, 2014.

[63] J. A. Lorenzen, B. B. Bonacci, R. E. Palmer et al., "Rbm19 is a nucleolar protein expressed in crypt/progenitor cells of the intestinal epithelium," Gene Expression Patterns, vol. 6, no. 1, pp. 45-56, 2005.

[64] M. Mak, F. Spill, R. D. Kamm, and M. H. Zaman, "Single-cell migration in complex microenvironments: mechanics and signaling dynamics," Journal of Biomechanical Engineering, vol. 138, no. 2, article 021004, 2016.

[65] Y. Yu, Y. Gao, H. Wang et al., "The matrix protein CCN1 (CYR61) promotes proliferation, migration and tube formation of endothelial progenitor cells," Experimental Cell Research, vol. 314, no. 17, pp. 3198-3208, 2008.

[66] E. C. K. Heng, Y. Huang, S. A. Black, and P. C. Trackman, "CCN2, connective tissue growth factor, stimulates collagen deposition by gingival fibroblasts via module 3 and $\alpha 6$ - and $\beta 1$ integrins," Journal of Cellular Biochemistry, vol. 98, no. 2, pp. 409-420, 2006.

[67] N. Chen, C. C. Chen, and L. F. Lau, "Adhesion of human skin fibroblasts to Cyr61 is mediated through integrin $\alpha_{6} \beta_{1}$ and cell surface heparan sulfate proteoglycans," Journal of Biological Chemistry, vol. 275, no. 32, pp. 24953-24961, 2000.

[68] T. M. Grzeszkiewicz, V. Lindner, N. Chen, S. C. T. Lam, and L. F. Lau, "The angiogenic factor cysteine-rich 61 (CYR61, CCN1) supports vascular smooth muscle cell adhesion and stimulates chemotaxis through integrin $\alpha 6 \beta 1$ and cell surface heparan sulfate proteoglycans," Endocrinology, vol. 143, no. 4, pp. 1441-1450, 2002.
[69] J. M. Schober, N. Chen, T. M. Grzeszkiewicz et al., "Identification of integrin $\alpha_{\mathrm{M}} \beta_{2}$ as an adhesion receptor on peripheral blood monocytes for Cyr61 (CCN1) and connective tissue growth factor (CCN2): immediate-early gene products expressed in atherosclerotic lesions," Blood, vol. 99, no. 12, pp. 4457-4465, 2002.

[70] F. Zhang, F. Hao, D. An et al., "The matricellular protein Cyr61 is a key mediator of platelet-derived growth factorinduced cell migration," The Journal of Biological Chemistry, vol. 290, no. 13, pp. 8232-8242, 2015.

[71] T. L. Huang, N. Mu, J. T. Gu et al., "DDR2-CYR61-MMP1 signaling pathway promotes bone erosion in rheumatoid arthritis through regulating migration and invasion of fibroblast-like synoviocytes," Journal of Bone and Mineral Research, vol. 32, no. 2, pp. 407-418, 2017.

[72] J. Wei, G. Yu, G. Shao et al., "CYR61 (CCN1) is a metastatic biomarker of gastric cardia adenocarcinoma," Oncotarget, vol. 7, no. 21, pp. 31067-31078, 2016.

[73] X. C. Li and J. L. Zhuo, "Recent updates on the proximal tubule renin-angiotensin system in angiotensin II-dependent hypertension," Current Hypertension Reports, vol. 18, no. 8, p. 63, 2016.

[74] J. Vian, C. Pereira, V. Chavarria et al., "The renin-angiotensin system: a possible new target for depression," BMC Medicine, vol. 15, no. 1, p. 144, 2017.

[75] S. Perez-Lloret, M. Otero-Losada, J. E. Toblli, and F. Capani, "Renin-angiotensin system as a potential target for new therapeutic approaches in Parkinson's disease," Expert Opinion on Investigational Drugs, vol. 26, no. 10, pp. 1163-1173, 2017.

[76] M. Holappa, H. Vapaatalo, and A. Vaajanen, "Many faces of renin-angiotensin system - focus on eye," The Open Ophthalmology Journal, vol. 11, no. 1, pp. 122-142, 2017.

[77] M. Pinter and R. K. Jain, “Targeting the renin-angiotensin system to improve cancer treatment: implications for immunotherapy," Science Translational Medicine, vol. 9, no. 410, p. eaan5616, 2017.

[78] E. Cigola, J. Kajstura, B. Li, L. G. Meggs, and P. Anversa, "Angiotensin II activates programmed myocyte cell death in vitro," Experimental Cell Research, vol. 231, no. 2, pp. 363-371, 1997.

[79] M. Urushihara and S. Kagami, "Role of the intrarenal reninangiotensin system in the progression of renal disease," Pediatric Nephrology, vol. 32, no. 9, pp. 1471-1479, 2017.

[80] Q. Feng, C. Lu, L. Wang, L. Song, C. Li, and R. C. Uppada, "Effects of renal denervation on cardiac oxidative stress and local activity of the sympathetic nervous system and reninangiotensin system in acute myocardial infracted dogs," BMC Cardiovascular Disorders, vol. 17, no. 1, p. 65, 2017.

[81] D. Xiong, W. Hu, S. T. Ye, and Y. S. Tan, "Isoliquiritigenin alleviated the Ang II-induced hypertensive renal injury through suppressing inflammation cytokines and oxidative stress-induced apoptosis via Nrf2 and NF- $\kappa$ B pathways," Biochemical and Biophysical Research Communications, vol. 506, no. 1, pp. 161-168, 2018.

[82] A. Hilfiker, D. Hilfiker-Kleiner, M. Fuchs et al., "Expression of CYR61, an angiogenic immediate early gene, in arteriosclerosis and its regulation by angiotensin II," Circulation, vol. 106, no. 2, pp. 254-260, 2002.

[83] D. Jacques, C. Provost, A. Normand, N. Abou Abdallah, J. Al-Khoury, and G. Bkaily, "Angiotensin II induces 
apoptosis of human right and left ventricular endocardial endothelial cells by activating the AT2 receptor," Canadian Journal of Physiology and Pharmacology, 2019.

[84] Z. Gao, X. Chen, K. Zhu, P. Zeng, and G. Ding, "Dab1 contributes to angiotensin II-induced apoptosis via p38 signaling pathway in podocytes," BioMed Research International, vol. 2017, Article ID 2484303, 11 pages, 2017.

[85] Y. P. Chen, K. Sivalingam, M. A. Shibu et al., "Protective effect of Fisetin against angiotensin II-induced apoptosis by activation of IGF-IR-PI3K-Akt signaling in H9c2 cells and spontaneous hypertension rats," Phytomedicine, vol. 57, pp. 1-8, 2018. 


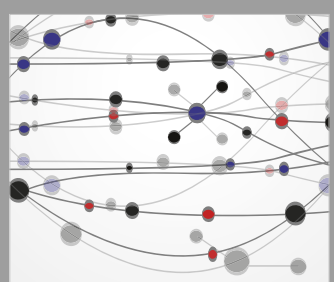

The Scientific World Journal
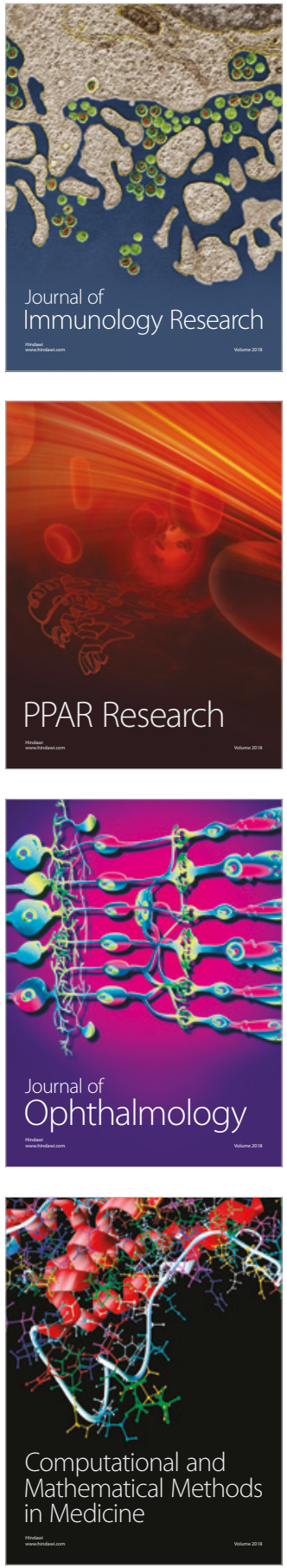

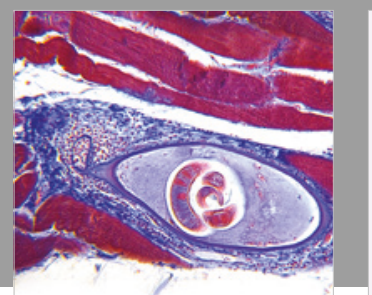

Gastroenterology Research and Practice

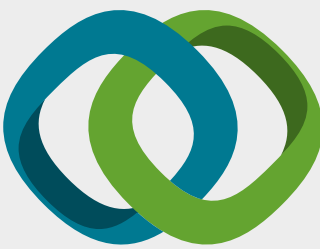

\section{Hindawi}

Submit your manuscripts at

www.hindawi.com
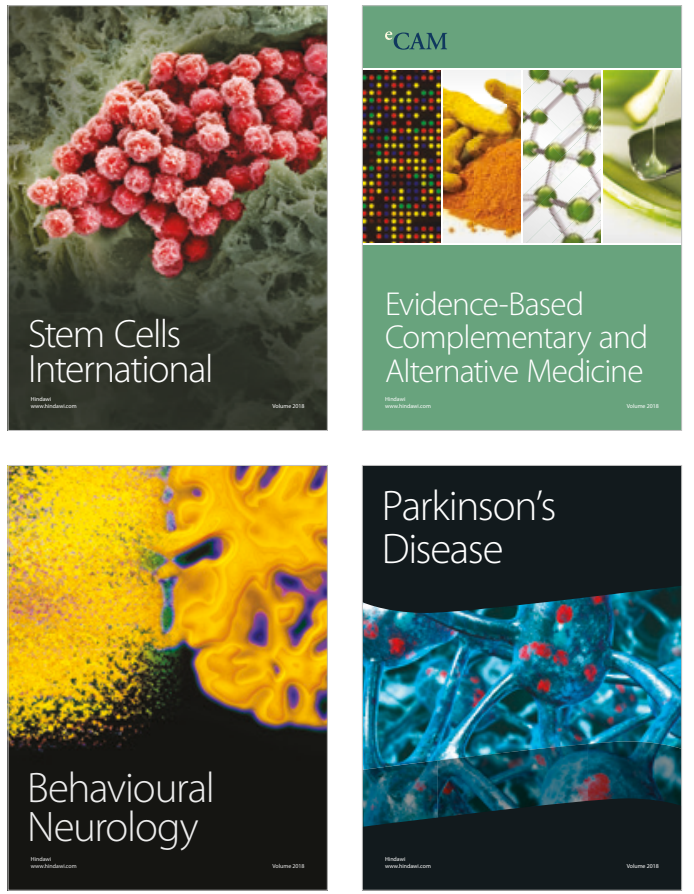

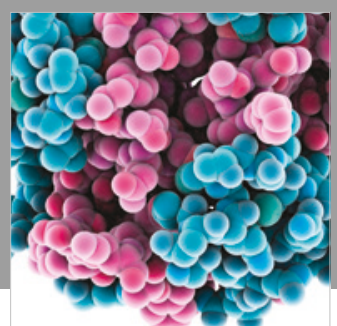

ournal of

Diabetes Research

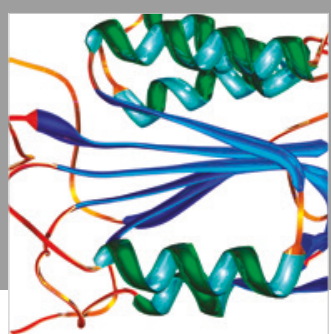

Disease Markers
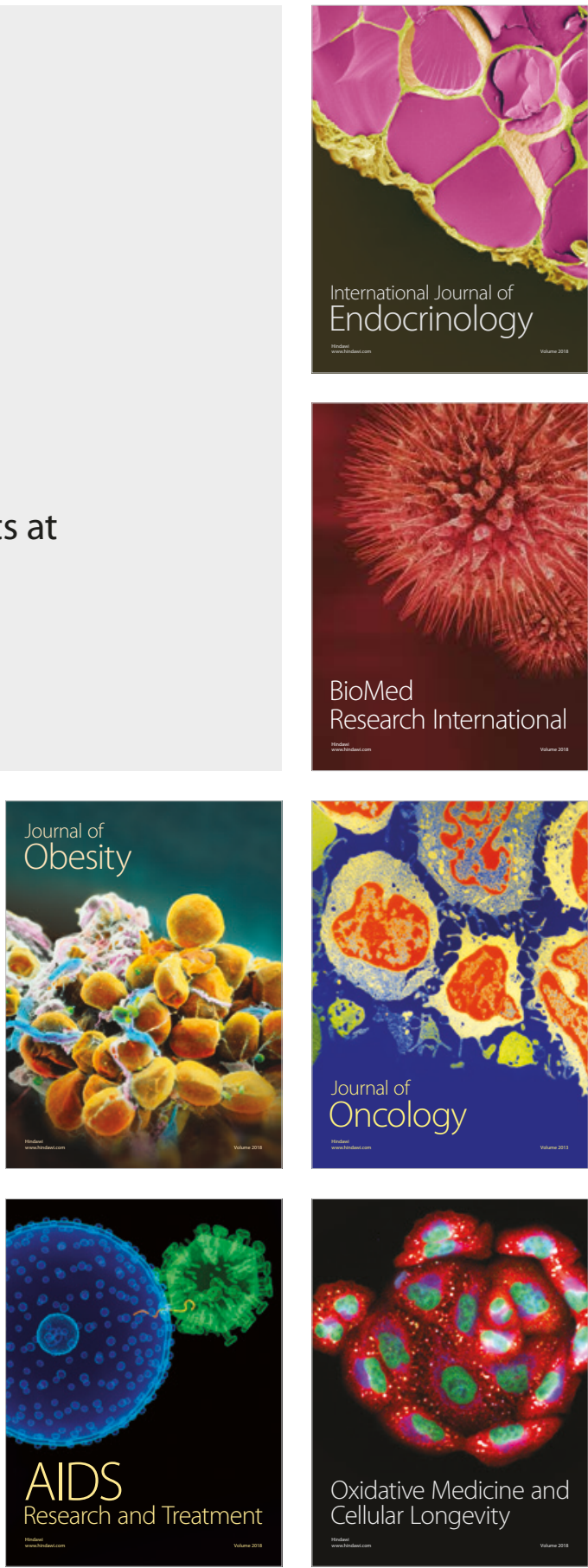